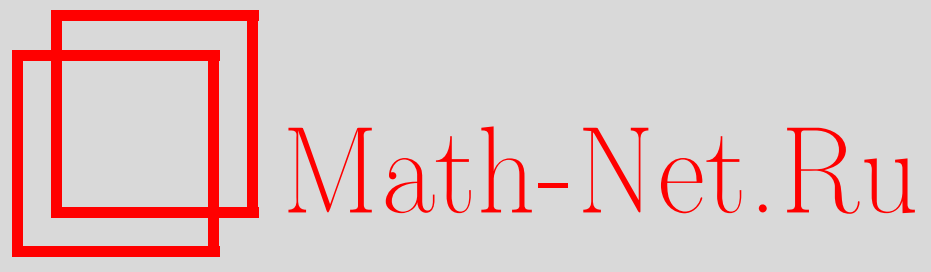

А. С. Юдина, Сингулярно возмущенное уравнение Бесселя в комплексных областях, Изв. РАН. Сер. матем., 2009, том 73, выпуск 3, 199-224

DOI: https://doi.org/10.4213/im2672

Использование Общероссийского математического портала Math-Net.Ru подразумевает, что вы прочитали и согласны с пользовательским соглашением http://www . mathnet.ru/rus/agreement

Параметры загрузки:

IP : 54.196 .121 .252

26 апреля 2023 г., 16:48:37

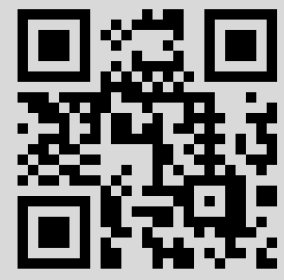


УДК 517.923

А. С. Юдина

\title{
Сингулярно возмущенное уравнение Бесселя в комплексных областях
}

\begin{abstract}
Для фундаментальной системы решений уравнения Бесселя методом регуляризации построены два вида регуляризованных асимптотических решений по комплексному параметру, первый - в замкнутой комплексной плоскости независимой переменной, за исключением особых точек функций спектра исходного оператора. Определены области равномерной и неравномерной асимптотической сходимости рядов в решениях. Проведено исследование полученных формул на действительной положительной оси, при этом доказано, что на интервале $(0,1)$, входящем в область неравномерной сходимости рядов, получаются известные асимптотические разложения Дебая для функций Бесселя. Второй вид регуляризованных равномерных асимптотических решений построен в окрестности регулярной особой точки из другой области изменения параметра уравнения. С использованием этих результатов получено равномерное асимптотическое решение краевой задачи для неоднородного и однородного уравнений Бесселя.
\end{abstract}

Библиография: 15 наименований.

Ключевые слова: уравнение Бесселя, регуляризирующая функция, регуляризованные асимптотические разложения, разложение Дебая, линии Стокса.

\section{Введение}

В работе применяется метод регуляризации для асимптотического интегрирования сингулярно возмущенных задач [1] в области дифференциальных уравнений с особыми точками.

Рассматривается уравнение Бесселя [2],[3]

$$
z^{2} w^{\prime \prime}+z w^{\prime}-\nu^{2}\left(1-z^{2}\right) w=0
$$

при комплексных $z$ и $\nu \rightarrow \infty$, для которого $z=0$ - регулярная особая точка (о. т.) и $z=\infty$ иррегулярная о. т. [4].

Уравнение $(0.1)$ заменой $\nu=1 / \varepsilon$ при $\varepsilon \rightarrow 0$ сводится к сингулярно возмущенному уравнению, к которому применяется метод регуляризации.

Настоящая статья является продолжением работ автора в области специальных функций и уравнений с особыми точками (см., например, [5]-[8]). В [7] был использован метод $\Phi$. Ольвера для дифференциальных уравнений специального вида. В [9]-[12] применялась идея метода регуляризации с использованием спектра промежуточного оператора и корней определяющего уравнения, что позволило получить ранее неизвестные асимптотические решения фундаментальных систем, сингулярно зависящие от параметра, и, как частный случай, новые асимптотические разложения функций Бесселя в окрестности регулярной о. т. 
В статье применен общий принцип метода регуляризации (без определяющего уравнения) с переходом в пространство большей размерности и использованием спектральных характеристик исходного оператора, что дало возможность получить единым методом два вида регуляризованных асимптотических при $\varepsilon \rightarrow 0$ решений для фундаментальной системы решений (ФСР) уравнения Бесселя не только в окрестности регулярной особой точки, а во всей замкнутой плоскости $\overline{\mathbb{C}}(z)$, за исключением особых точек $z=0, z=-1, z=1$ функций спектра оператора Бесселя.

В полученных формулах регуляризованных ФСР доказана асимптотическая сходимость регулярных рядов при $\varepsilon \rightarrow 0$ с указанием областей в плоскости $\varepsilon$ и с указанием областей равномерной и неравномерной асимптотической сходимости рядов по $z$.

Исследованы полученные формулы для ФСР на положительной действительной оси. Произведено сравнение полученных результатов с ранее известными асимптотическими разложениями функций Бесселя. На интервале $(0,1)$ из формул следуют асимптотические разложения Дебая для функций Бесселя [3, c. 187]. С использованием полученных результатов методом регуляризации построено равномерное асимптотическое решение краевой задачи в окрестности регулярной о. т. для неоднородного и однородного уравнений Бесселя.

\section{$\S 1$. Структура фундаментальной системы решений сингулярно возмущенного уравнения Бесселя в комплексных областях $z$ и $\varepsilon$}

В уравнении (0.1) сделаем замену $\nu=1 / \varepsilon$ и получим

$$
L_{\varepsilon} w \equiv \varepsilon^{2} z^{2} w^{\prime \prime}+\varepsilon^{2} z w^{\prime}-\left(1-z^{2}\right) w=0 .
$$

Это сингулярно возмущенное уравнение по параметру $\varepsilon$ при $\varepsilon \rightarrow 0$ с особыми точками $z=0$ и $z=\infty$ по независимой переменной.

1.1. Формализм метода регуляризации. Для построения ФСР уравнения (1.1) определим в замкнутой плоскости $\overline{\mathbb{C}}(z)$ область стабильности спектра исходного оператора $L_{\varepsilon}[1$, гл. 2].

Из характеристического уравнения

$$
z^{2} \lambda^{2}(z)-\left(1-z^{2}\right)=0
$$

определим функции спектра

$$
\lambda_{1}(z)=+\frac{\sqrt{1-z^{2}}}{z}, \quad \lambda_{2}(z)=-\frac{\sqrt{1-z^{2}}}{z},
$$

для корня берется та ветвь, которая при $z=0$ равна единице.

Исследуем функции (1.3). Особая точка $z=0$ уравнения (1.1) является особой и для функций $\lambda_{s}(z), s=1,2$; точка $z=\infty$ не является особой для $\lambda_{s}(z), \lim _{z \rightarrow \infty} \lambda_{s}(z)=(-1)^{s-1} i$. Пусть $\lambda_{s}(\infty)=(-1)^{s-1} i$ и $\lambda_{s}(z)-$ непрерывные функции в $\overline{\mathbb{C}}(z) \backslash\{z=0\}$. Производная в точке $z=\infty$ не имеет смысла, но принято принимать функции $\lambda_{s}(z)$ аналитическими при $z=\infty$, так как $\lambda_{s}(1 / z)=(-1)^{s-1} \sqrt{z^{2}-1}=\widetilde{\lambda}_{s}(z)-$ аналитические функции в точке $z=0$. 
Следовательно:

1) $\lambda_{s}(z), s=1,2,-$ аналитические функции в области

$$
\bar{G}(z)=\left\{z: z \in \overline{\mathbb{C}}(z) \backslash\left\{z_{1}=0, z_{2}=-1, z_{3}=1\right\}\right\} ;
$$

2) $\lambda_{1}(z) \neq \lambda_{2}(z), \lambda_{s}(z) \neq 0$ в $\bar{G}(z)$, поэтому будем применять метод регуляризации к уравнению $(1.1)$ в области $\bar{G}(z),(1.4)$, в которой выполняются условия стабильности спектра $1^{0}, 2^{0}$ из $[1$, с. 39$]$.

Для определения каждого решения $w_{s}(z, \varepsilon), s=1,2$, ФСР вводим соответствующую регуляризирующую функцию $\varphi_{s}(z, \varepsilon)$ через функцию спектра $\lambda_{s}(z)$, которую наряду с независимой переменной $z \in \bar{G}(z)$ принимаем за новую независимую переменную $\tau_{s}$ :

$$
\tau_{s}=\frac{1}{\varepsilon} \int_{z_{0}}^{z} \lambda_{s}(\xi) d \xi \equiv \varphi_{s}(z, \varepsilon), \quad s=1,2, \quad z_{0} \neq 0,
$$

первообразная $F_{s}(z)$ для $\lambda_{s}(z)$ имеет вид

$$
F_{s}(z)=(-1)^{s-1}\left[\sqrt{1-z^{2}}+\ln z-\ln \left(1+\sqrt{1-z^{2}}\right)\right] .
$$

Точка $z_{0}$ определяет постоянный множитель в решениях $w_{s}(z, \varepsilon)$.

Далее в настоящем пункте для сокращения записей нижний индекс $s$ мы не используем, имея в виду, что рассматривается случай $s=1$ или $s=2$.

Вместо искомого решения $w(z, \varepsilon)$ уравнения (1.1) будем изучать расширенную функцию $\widetilde{w}(z, \tau, \varepsilon)$ такую, что ее сужение при $\tau=\varphi(z, \varepsilon)$ совпадает c $w(z, \varepsilon): \widetilde{w}(z, \varphi(z, \varepsilon), \varepsilon) \equiv w(z, \varepsilon)$.

Определим из уравнения (1.1) уравнение для функции $\widetilde{w}(z, \tau, \varepsilon)$ :

$$
L_{\varepsilon} \widetilde{w} \equiv \varepsilon^{2} L_{2} \widetilde{w}+\varepsilon L_{1} \widetilde{w}+L_{0} \widetilde{w}=0,
$$

где

$$
\begin{gathered}
L_{0} \widetilde{w} \equiv z^{2} \lambda^{2}(z) \frac{\partial^{2} \widetilde{w}}{\partial \tau^{2}}-\left(1-z^{2}\right) \widetilde{w}, \quad L_{1} \widetilde{w} \equiv 2 z^{2} \lambda(z) \frac{\partial^{2} \widetilde{w}}{\partial z \partial \tau}+z(z \lambda(z))^{\prime} \frac{\partial \widetilde{w}}{\partial \tau}, \\
L_{2} \widetilde{w} \equiv z^{2} \frac{\partial^{2} \widetilde{w}}{\partial z^{2}}+z \frac{\partial \widetilde{w}}{\partial z}
\end{gathered}
$$

Решение уравнения (1.6) определяем в виде ряда:

$$
\widetilde{w}(z, \tau, \varepsilon)=\sum_{k=0}^{\infty} \varepsilon^{k} w_{k}(z, \tau) .
$$

Для коэффициентов ряда (1.7) из уравнения (1.6) получаем следующие рекуррентные уравнения:

$$
\begin{gathered}
L_{0} w_{0}(z, \tau)=0, \quad L_{0} w_{1}(z, \tau)=-L_{1} w_{0}(z, \tau), \\
L_{0} w_{k}(z, \tau)=-L_{1} w_{k-1}(z, \tau)-L_{2} w_{k-2}(z, \tau), \quad k=2,3, \ldots
\end{gathered}
$$

Для решения уравнений (1.8) вводим пространство безрезонансных решений

$$
Y=\left\{w_{k}: w_{k}(z, \tau)=e^{\tau} D_{k}(z), k=0,1, \ldots\right\} .
$$


Определим результаты действия операторов $L_{j}, j=0,1,2$, из (1.6) на функции $w_{k}(z, \tau) \in Y$. На элементах пространства $Y$ основной оператор $L_{0}$ имеет вид

$$
L_{0} w_{k}(z, \tau) \equiv\left[z^{2} \lambda^{2}(z)-\left(1-z^{2}\right)\right] D_{k}(z) e^{\tau} \equiv 0
$$

для всех $k=0,1, \ldots$, так как $\lambda(z)$ - корень уравнения (1.2),

$$
\begin{gathered}
L_{1} w_{k}(z, \tau) \equiv\left[2 z \lambda(z) D_{k}^{\prime}(z)+(z \lambda(z))^{\prime} D_{k}(z)\right] z e^{\tau}, \\
L_{2} w_{k}(z, \tau) \equiv\left[z D_{k}^{\prime \prime}(z)+D_{k}^{\prime}(z)\right] z e^{\tau} .
\end{gathered}
$$

В (1.11) введем операторы $\tilde{L}_{i}, i=1,2$, следующим образом:

$$
\begin{gathered}
\tilde{L}_{1} D_{k}(z) \equiv 2 z \lambda(z) D_{k}^{\prime}(z)+(z \lambda(z))^{\prime} D_{k}(z), \\
\tilde{L}_{2} D_{k}(z) \equiv z D_{k}^{\prime \prime}(z)+D_{k}^{\prime}(z) .
\end{gathered}
$$

Из (1.8)-(1.12) получаем для определения функций $D_{k}(z)$ рекуррентные линейные уравнения первого порядка:

$$
\tilde{L}_{1} D_{0}(z)=0, \quad \tilde{L}_{1} D_{k+1}(z)=-\tilde{L}_{2} D_{k}(z), \quad k=0,1, \ldots
$$

Функции $D_{k}(z)$, определенные из уравнений (1.13) (конкретно это будет сделано в следующем пункте), подставляем в (1.9) и, далее, в ряд (1.7). В результате будем иметь формальное решение уравнения (1.6) в виде

$$
\widetilde{w}(z, \tau, \varepsilon)=e^{\tau} \sum_{k=0}^{\infty} \varepsilon^{k} D_{k}(z) .
$$

Проведя сужение решения (1.14) при $\tau=\varphi(z, \varepsilon),(1.5)$, получаем каждое формальное решение $\Phi С Р$ уравнения (1.1) в области $\bar{G}(z)$ в виде

$$
w(z, \varepsilon)=\exp \left\{\frac{1}{\varepsilon} \int_{z_{0}}^{z} \lambda(\xi) d \xi\right\} \sum_{k=0}^{\infty} \varepsilon^{k} D_{k}(z) .
$$

ОПРЕДЕЛЕНИЕ 1.1. Решение $w(z, \varepsilon)$ вида (1.15), в котором сингулярная зависимость от $\varepsilon$ выделяется экспоненциальным множителем перед регулярным рядом по асимптотической последовательности $\left\{\varepsilon^{n}\right\}$ при $\varepsilon \rightarrow 0$, будем называть формальным регуляризованным решением.

1.2. Формальная регуляризованная ФСР уравнения (1.1) в области $\bar{G}(z)$. В формуле (1.15) для ФСР введем индекс $s$ :

$$
w_{s}(z, \varepsilon)=\exp \left\{\frac{1}{\varepsilon} \int_{z_{0}}^{z} \lambda_{s}(\xi) d \xi\right\} \sum_{k=0}^{\infty} \varepsilon^{k} D_{k}^{(s)}(z), \quad s=1,2,
$$

где $D_{k}^{(s)}(z)$ определяются из соотношений $(1.12),(1.13)$.

Запишем рекуррентную систему (1.13) для $\lambda_{1}(z), s=1$, в развернутом виде, обозначив $D_{k}^{(1)}(z)=D_{k}(z)$ :

$$
\begin{gathered}
2 \sqrt{1-z^{2}} D_{0}^{\prime}(z)-\frac{z}{\sqrt{1-z^{2}}} D_{0}(z)=0 \\
2 \sqrt{1-z^{2}} D_{k+1}^{\prime}(z)-\frac{z}{\sqrt{1-z^{2}}} D_{k+1}(z)=-\left(z D_{k}^{\prime}(z)\right)^{\prime} .
\end{gathered}
$$


Из системы (1.17) нетрудно сделать вывод, что $D_{k}^{(2)}(z)=(-1)^{k} D_{k}^{(1)}(z)$, так как $\lambda_{2}(z)=-\lambda_{1}(z)$.

Система (1.17) имеет особые точки $z= \pm 1$, совпадающие с о. т. функций спектра. Особые точки уравнения (1.1) не оказывают влияния на регулярные ряды в (1.16), коэффициенты ряда $D_{k}^{(s)}(z)$ будут определяться из (1.17) в области

$$
G^{*}(z)=\{z: z \in \overline{\mathbb{C}}(z) \backslash\{z= \pm 1\}\}, \quad G^{*}(z) \supset \bar{G}(z) .
$$

Соотношение (1.18) отражает существенный факт при применении метода регуляризации к уравнению Бесселя: регулярные ряды по $\varepsilon$ в формуле (1.16) определены в более широкой области, чем область стабильности спектра.

Из первого уравнения (1.17) находим

$$
D_{0}(z)=\frac{C}{\sqrt[4]{1-z^{2}}},
$$

где $C$ - произвольная постоянная. Для построения $\Phi С Р$ принимаем $C=1$ и запишем

$$
D_{0}(z)=\frac{1}{\sqrt[4]{1-z^{2}}}
$$

Следующие уравнения в (1.17) решаем методом вариации произвольной постоянной, принимая

$$
D_{k+1}(z)=D_{0}(z) C_{k+1}(z), \quad k=0,1,2, \ldots
$$

Для определения функций $C_{k+1}(z)$ получаем уравнения

$$
C_{k+1}^{\prime}(z)=-\frac{\left(z D_{k}^{\prime}(z)\right)^{\prime}}{2 \sqrt[4]{1-z^{2}}}, \quad k=0,1, \ldots
$$

В уравнениях (1.21) от переменной $z$ перейдем к переменной $t$ по формуле

$$
t=\frac{1}{\sqrt{1-z^{2}}}, \quad t(0)=+1 .
$$

Функция $t(z)$ является аналитической в области $G^{*}(z)$ и переводит точки $z=-1$ и $z=1$ в $t=\infty$, точка $z=\infty$ переходит в $t=0$, а точка $z=0-$ в $t=1$, область $G^{*}(z),(1.18)$, переходит в область $T(t)$.

При переходе к переменной $t$ система (1.21) перейдет в систему, не имеющую особых точек в области $T$, и при ее решении выявится структура функций $D_{k}(z)$. Будет справедлива следующая

ТЕОРема 1.1. Формальная регуляризованная ФСР, (1.16), уравнения (1.1) в области $\bar{G}(z),(1.4)$, имеет вид

$$
w_{s}(z, \varepsilon)=\exp \left\{\frac{1}{\varepsilon} \int_{z_{0}}^{z} \lambda_{s}(\xi) d \xi\right\} \sqrt{t} \sum_{k=0}^{\infty} \varepsilon^{k} u_{k}^{(s)}(t), \quad t=\frac{1}{\sqrt{1-z^{2}}}
$$

Коэффициенты ряда $u_{k}^{(1)}(t)=u_{k}(t)$ в (1.23) при $s=1$ определяются в области

$$
T=\{t: t \in \mathbb{C}, t(0)=+1\}
$$


по рекуррентной формуле

$$
u_{0}(t)=1, \quad u_{k+1}(t)=\left.\frac{1}{2} t^{2}\left(1-t^{2}\right) u_{k}^{\prime}(t)\right|_{t_{0}} ^{t}+\frac{1}{8} \int_{t_{0}}^{t}\left(1-5 \zeta^{2}\right) u_{k}(\zeta) d \zeta
$$

при любом фиксированном $t_{0} \in T$ и являются многочленами по $t$ степени $3 k$, $u_{k}^{(2)}(t)=(-1)^{k} u_{k}^{(1)}(t)=(-1)^{k} u_{k}(t)$.

ДокАЗАТЕЛЬство. Перейдем к переменной $t$ в функциях $D_{k}(z), k=0,1, \ldots$, (1.19), (1.20):

$$
D_{0}(z)=\widetilde{D}_{0}(t)=\sqrt{t}, \quad D_{k+1}(z)=\widetilde{D}_{k+1}(t)=\sqrt{t} \widetilde{C}_{k+1}(t),
$$

где обозначим

$$
\widetilde{C}_{k+1}(t)=u_{k+1}(t), \quad k=0,1, \ldots, \quad u_{0}(t)=1 .
$$

Тогда из (1.26) имеем

$$
D_{0}(z)=\sqrt{t}, \quad D_{k+1}(z)=\sqrt{t} u_{k+1}(t), \quad k=0,1, \ldots .
$$

Подставим (1.28) в ряд (1.16), и формула (1.16) для ФСР примет вид (1.23).

Из $(1.28),(1.17)$ будут следовать соотношения $u_{k}^{(2)}(t)=(-1)^{k} u_{k}^{(1)}(t)$, так как $D_{k}^{(2)}(z)=(-1)^{k} D_{k}^{(1)}(z)$.

Перейдем к доказательству формулы (1.25). Уравнения (1.21) при переходе к переменной $t$ в силу (1.27) примут вид

$$
u_{k+1}^{\prime}(t)=-\frac{1}{2} \sqrt{t}\left[\left(t^{3}-t\right) \widetilde{D}_{k}^{\prime}(t)\right]^{\prime}, \quad k=0,1, \ldots
$$

Из $(1.26),(1.27)$ справедливо соотношение $\sqrt{t} \widetilde{D}_{k}^{\prime}(t)=\frac{1}{2} u_{k}(t)+t u_{k}^{\prime}(t)$, учитываем его в соотношении (1.29):

$$
u_{k+1}^{\prime}(t)=-\frac{1}{2} \sqrt{t}\left[\frac{1}{2}\left(t^{5 / 2}-t^{1 / 2}\right) u_{k}(t)+\left(t^{7 / 2}-t^{3 / 2}\right) u_{k}^{\prime}(t)\right]^{\prime},
$$

в результате получим для определения функций $u_{k+1}(t), k=0,1, \ldots$, рекуррентные уравнения первого порядка, определенные в области $T$. Для однозначного решения каждого уравнения (1.30) задаем задачи Коши при любом фиксированном $t_{0} \in T$ с условиями $u_{k+1}\left(t_{0}\right)=0$, из (1.30) получаем функции $u_{k+1}(t)$, определяемые по рекуррентной формуле

$u_{0}(t)=1, \quad u_{k+1}(t)=-\frac{1}{2} \int_{t_{0}}^{t} \sqrt{\zeta}\left[\frac{1}{2}\left(\zeta^{5 / 2}-\zeta^{1 / 2}\right) u_{k}(\zeta)+\left(\zeta^{7 / 2}-\zeta^{3 / 2}\right) u_{k}^{\prime}(\zeta)\right]^{\prime} d \zeta$.

Интегрируем в (1.31) и получаем формулу (1.25), из которой следует, что функции $u_{k}(t)$ суть многочлены по $t$ степени $3 k$. Теорема доказана.

В настоящей работе рассмотрим два возможных случая в формуле (1.25):

$1)$ случай $t_{0}=0$, соответствующий особой точке $z=\infty$ уравнения (1.1);

2) случай $t_{0}=1$, соответствующий особой точке $z=0$.

Для каждого случая проведено доказательство асимптотической сходимости рядов при $\varepsilon \rightarrow 0$.

ОПРЕДЕЛЕНИЕ 1.2. Если в формуле (1.23) для $w_{s}(z, \varepsilon), s=1$ или $s=2$, доказана асимптотическая сходимость регулярных рядов при $\varepsilon \rightarrow 0$, то формулу (1.23) будем называть регуляризованным асимптотическим решением. 
1.3. Регуляризованная асимптотическая ФСР уравнения (1.1) в области $\bar{G}(z)$. Будем определять решения $w_{s}(z, \varepsilon)$ по формуле (1.23), принимая $t_{0}=0$ для определения коэффициентов рядов в формуле (1.25), которая при $s=1, u_{k}^{(1)}(t)=u_{k}(t)$ будет иметь вид

$u_{0}(t)=1, \quad u_{k+1}(t)=\frac{1}{2} t^{2}\left(1-t^{2}\right) u_{k}^{\prime}(t)+\frac{1}{8} \int_{0}^{t}\left(1-5 \zeta^{2}\right) u_{k}(\zeta) d \zeta, \quad k=0,1, \ldots$

Из (1.32) следует, что в этом случае многочлены $u_{k}(t)$ имеют $k+1$ членов от $t^{k}$ до $t^{3 k}$, четных при четном $k$ и нечетных при нечетном $k, u_{k}^{(2)}(t)=$ $(-1)^{k} u_{k}^{(1)}(t)$.

Обозначим

$$
u_{k}(t)=\sum_{j=0}^{k} \alpha_{k, k+2 j} t^{k+2 j}, \quad k=1,2, \ldots
$$

Первые $u_{k}(t), k=1,2$, имеют вид

$$
u_{1}(t)=\frac{1}{24}\left(3 t-5 t^{3}\right), \quad u_{2}(t)=\frac{1}{1152}\left(81 t^{2}-462 t^{4}+385 t^{6}\right)
$$

(при $k=1,2,3,4$ см. [3, с. 187]).

Для доказательства асимптотической сходимости рядов в (1.23) при $t_{0}=0$ запишем формулу (1.23) для ФСР в виде

$$
w_{s}(z, \varepsilon)=\exp \left\{\frac{1}{\varepsilon} \int_{z_{0}}^{z} \lambda_{s}(\xi) d \xi\right\} \sqrt{t}\left(1+R_{1}^{(s)}(t, \varepsilon)\right), \quad s=1,2,
$$

где

$$
R_{1}^{(s)}(t, \varepsilon)=\sum_{k=1}^{\infty} \varepsilon^{k} u_{k}^{(s)}(t), \quad u_{k}^{(2)}(t)=(-1)^{k} u_{k}^{(1)}(t) .
$$

Ряды в (1.35) являются формальными рядами по асимптотической последовательности $\left\{\varepsilon_{k}\right\}, k=1,2, \ldots$, при $\varepsilon \rightarrow 0$ для функций $R_{1}^{(s)}(t, \varepsilon)$, определенных из формулы (1.34).

ЛЕмма 1.1. 1) В ряде из (1.35) для коэффициентов $u_{k}^{(s)}(t), k=1,2, \ldots$, определяемых по формуле (1.32), справедливы следующие неравенства:

$$
\left|u_{k}^{(s)}(t)\right| \leqslant \begin{cases}4^{k}(k-1) ! & \text { nрu }|t| \leqslant 1 \\ \left(4|t|^{3}\right)^{k}(k-1) ! & \text { npu }|t|>1\end{cases}
$$

2) Ряды в (1.35) являются асимптотическими разложениями функиий $R_{1}^{(s)}(t, \varepsilon), s=1,2$, при $\varepsilon=\operatorname{Re}^{i \varphi}, \varepsilon \rightarrow 0$, по лучам ${ }^{1}$ в области $G_{1}(\varepsilon)=\{\varepsilon: 0<$ $|\varepsilon|<1 / 4\}$, равномерными по $t$ nри $|t| \leqslant 1$ и неравномерными по $t$ nри $|t|>1$ в области $G_{2}(\varepsilon, t)=\left\{\varepsilon: 0<|\varepsilon|<1 /\left(4|t|^{3}\right)\right\}$.

ДоказАтельство. 1) Из (1.35) следует, что для доказательства формулы (1.36) достаточно рассмотреть случай $s=1, u_{k}^{(1)}(t)=u_{k}(t)$.

Многочлены $u_{k}(t), k=1,2, \ldots$, определяются по рекуррентной формуле (1.32).

\footnotetext{
${ }^{1}$ Далее будем записывать $\varepsilon \rightarrow 0$ (по $R$ ).
} 
Рассмотрим случай $k=0, k+1=1$. Из (1.32), (1.33) имеем

$$
\begin{gathered}
u_{1}(t)=\frac{1}{8} t-\frac{5}{24} t^{3}=\alpha_{11} t+\alpha_{13} t^{3}, \quad\left|\alpha_{11}\right|+\left|\alpha_{13}\right|<4, \\
\left|u_{1}(t)\right| \leqslant \begin{cases}\left|\alpha_{11}\right|+\left|\alpha_{13}\right| \leqslant 4 \cdot 0 ! & \text { при }|t| \leqslant 1, \\
|t|^{3}\left(\left|\alpha_{11}\right|+\left|\alpha_{13}\right|\right) \leqslant\left|t^{3}\right| \cdot 4 \cdot 0 ! & \text { при }|t|>1 .\end{cases}
\end{gathered}
$$

Получили формулу (1.36) при $k=1$.

Вывод оценки для $\left|u_{2}(t)\right|$ изложим подробно. Имея $u_{1}(t)=\alpha_{11} t+\alpha_{13} t^{3}=$ $\alpha_{11} t^{k}+\alpha_{13} t^{3 k}$ и применяя (1.33), (1.32), при $k=1, k+1=2$, получим

$$
\begin{aligned}
u_{2}(t)= & \alpha_{22} t^{2}+\alpha_{24} t^{4}+\alpha_{26} t^{6} \\
= & \alpha_{11}\left(\frac{1}{2} k+\frac{1}{8(k+1)}\right) t^{2}+\left[\alpha_{11}\left(-\frac{1}{2} k-\frac{5}{8(k+3)}\right)\right. \\
& \left.\quad+\alpha_{13}\left(\frac{1}{2} 3 k+\frac{1}{8(k+3)}\right)\right] t^{4}+\alpha_{13}\left(-\frac{1}{2} 3 k-\frac{5}{8 \cdot 3(k+1)}\right) t^{6} .
\end{aligned}
$$

Из (1.38) следуют неравенства

$$
\left|u_{2}(t)\right| \leqslant \begin{cases}\left|\alpha_{22}\right|+\left|\alpha_{24}\right|+\left|\alpha_{26}\right| & \text { при }|t| \leqslant 1 \\ \left(|t|^{3}\right)^{2}\left(\left|\alpha_{22}\right|+\left|\alpha_{24}\right|+\left|\alpha_{26}\right|\right) & \text { при }|t|>1\end{cases}
$$

где

$$
\begin{gathered}
\left|\alpha_{22}\right|=\left|\alpha_{11}\right|\left(\frac{1}{2} k+\frac{1}{8(k+1)}\right), \\
\left|\alpha_{24}\right| \leqslant\left|\alpha_{11}\right|\left(\frac{1}{2} k+\frac{5}{8(k+3)}\right)+\left|\alpha_{13}\right|\left(\frac{1}{2} 3 k+\frac{1}{8(k+3)}\right), \\
\left|\alpha_{26}\right|=\left|\alpha_{13}\right|\left(\frac{1}{2} 3 k+\frac{5}{8 \cdot 3(k+1)}\right) .
\end{gathered}
$$

В (1.40) наибольшим множителем при $\left|\alpha_{1,1+2 j}\right|, j=0,1$, будет множитель при $\left|\alpha_{13}\right|$ в выражении для $\left|\alpha_{26}\right|$. Оценим его:

$$
\left(\frac{1}{2} 3 k+\frac{5}{8 \cdot 3(k+1)}\right)<\left(\frac{3 k}{2}+\frac{1}{2}\right)=\frac{1}{2}(3 k+1) \leqslant 2 k, \quad k \geqslant 1 .
$$

Применяем (1.41) к неравенствам (1.40), последние примут вид

$$
\left|\alpha_{22}\right| \leqslant 2 k\left|\alpha_{11}\right|, \quad\left|\alpha_{24}\right| \leqslant 2 k\left(\left|\alpha_{11}\right|+\left|\alpha_{13}\right|\right), \quad\left|\alpha_{26}\right| \leqslant 2 k\left|\alpha_{13}\right| .
$$

Учитывая, что в формуле из (1.42) каждое $\left|\alpha_{1,1+2 j}\right|, j=0,1$, входит два раза, и применяя неравенства (1.37), получим следующую оценку при $k=1$ :

$$
\left|\alpha_{22}\right|+\left|\alpha_{24}\right|+\left|\alpha_{26}\right| \leqslant 4 k\left(\left|\alpha_{11}\right|+\left|\alpha_{13}\right|\right) \leqslant 4^{2} \cdot 1 ! .
$$

Подставляя (1.43) в (1.39), получим (1.36) при $k=2$ :

$$
\left|u_{2}(t)\right| \leqslant \begin{cases}4^{2} \cdot 1 ! & \text { при }|t| \leqslant 1 \\ 4^{2}\left(|t|^{3}\right)^{2} \cdot 1 ! & \text { при }|t|>1\end{cases}
$$


Рассмотрим общий случай в формуле (1.32). Предположим, что для функции $u_{k}(t)=\sum_{j=0}^{k} \alpha_{k, k+2 j} t^{k+2 j}$ справедливо неравенство, аналогичное (1.43) при $k=2$ :

$$
\sum_{j=0}^{k}\left|\alpha_{k, k+2 j}\right| \leqslant 4^{k}(k-1) !
$$

Для функции $u_{k+1}(t)=\sum_{j=0}^{k+1} \alpha_{k+1,(k+1)+2 j} t^{(k+1)+2 j}$ будет справедливо следующее:

$$
\left|u_{k+1}(t)\right| \leqslant \begin{cases}\sum_{j=0}^{k}\left|\alpha_{k+1,(k+1)+2 j}\right| & \text { при }|t| \leqslant 1, \\ \left(|t|^{3}\right)^{k+1} \sum_{j=0}^{k}\left|\alpha_{k+1,(k+1)+2 j}\right| & \text { при }|t|>1 .\end{cases}
$$

Далее, по формуле (1.32) получим выражение $u_{k+1}(t)$ через коэффициенты функции $u_{k}(t)$, аналогичные $(1.38)$ для $u_{2}(t)$.

Учитывая подробное доказательство формулы (1.36) для функции $u_{2}(t)$, простую структуру многочленов $u_{k}(t)$ и прозрачность формулы $(1.32)$, далее ограничимся фрагментарным доказательством.

Из формулы (1.32) очевидно, что функция $u_{k+1}(t)$ имеет $2(k+1)$ членов с коэффициентами функции $u_{k}(t)$ и каждый коэффициент входит два раза, причем наибольший по модулю множитель, зависящий от $k$, будет при члене с наибольшей степенью $t^{3(k+1)}$. Получим из $(1.32)$ этот коэффициент, он образован из двух слагаемых:

$$
-\frac{1}{2} t^{4}\left(\alpha_{k, 3 k} t^{3 k}\right)^{\prime}+\frac{1}{8} \int_{0}^{t}\left(-5 \zeta^{2}\right) \alpha_{k, 3 k} \zeta^{3 k} d \zeta=\alpha_{k, 3 k}\left(-\frac{1}{2} 3 k-\frac{5}{8} \frac{1}{3(k+1)}\right) t^{3(k+1)} .
$$

Сравнивая (1.46) с (1.38), легко видеть, что оценкой по модулю множителя в (1.46) будет оценка (1.41), справедливая для любых $k$.

Учитывая это, получим оценки для $\left|\alpha_{k+1,(k+1)+2 j}\right|, j=\overline{0, k+1}$, аналогичные (1.42), через модули коэффициентов $u_{k}(t)$, при $j=0$ и $j=k+1$ оценки имеют по одному слагаемому, а при остальных $j$ - по два слагаемых. Используя это, при выполнении (1.44) получим

$$
\sum_{j=0}^{k+1}\left|\alpha_{k+1,(k+1)+2 j}\right| \leqslant 4 k \sum_{j=0}^{k}\left|\alpha_{k, k+2 j}\right| \leqslant 4^{k+1} k ! .
$$

Подставляя (1.47) в (1.45), имеем

$$
\left|u_{k+1}(t)\right| \leqslant \begin{cases}4^{k+1} k ! & \text { при }|t| \leqslant 1 \\ \left(|t|^{3}\right)^{k+1} 4^{k+1} k ! & \text { при }|t|>1 .\end{cases}
$$

Неравенства (1.48) эквивалентны неравенствам (1.36). Утверждение 1) доказано.

2) Пусть $|t| \leqslant 1$. Из (1.35), (1.36) справедливы неравенства при $s=1,2$ для любых $|t| \leqslant 1$ :

$$
\left|R_{1}^{(s)}(t, \varepsilon)\right|=\left|\sum_{k=1}^{\infty} \varepsilon^{k} u_{k}^{(s)}(t)\right| \leqslant \sum_{k=1}^{\infty}\left|\varepsilon^{k}\right|\left|u_{k}^{(s)}(t)\right| \leqslant \sum_{k=1}^{\infty}(4|\varepsilon|)^{k}(k-1) ! .
$$


Обозначим $4|\varepsilon|=\frac{1}{x}$. При $x>1$ имеем $|\varepsilon|<\frac{1}{4}$ и запишем (1.49) в виде (заметив, что $|\varepsilon| \rightarrow 0$ при $x \rightarrow \infty)$

$$
\left|R_{1}^{(s)}(t, \varepsilon)\right| \leqslant \sum_{k=1}^{\infty} \frac{(k-1) !}{x^{k}}=\frac{1}{x}+\frac{1 !}{x^{2}}+\frac{2 !}{x^{3}}+\cdots+\frac{(k-1) !}{x^{k}}+\cdots .
$$

Далее индекс $s$ выписывать не будем, имея в виду $s=1,2$. В (1.50) находится положительный ряд по асимптотической последовательности $\left\{\frac{1}{x^{k}}\right\}, k=1,2 \ldots$, при $x \rightarrow \infty$, который по свойствам асимптотических рядов представляет не одну функцию, а класс асимптотически равных функций. Поэтому для оценки величины $\left|R_{1}(t, \varepsilon)\right|$ по (1.50) достаточно указать одну функцию $F(x)$, для которой ряд в (1.50) является асимптотическим разложением при $x \rightarrow \infty$, так как $O$-оценки остаточных членов ряда для всех функций, асимптотически равных $F(x)$, будут одинаковыми.

Такая функция $F(x)$ приведена в работе [14, с. 22]:

$$
F(x)=e^{-x} f(x), \quad f(x)=\int_{1}^{x} \frac{e^{t}}{t} d t=\operatorname{Ei}(x)-\operatorname{Ei}(1),
$$

где $\operatorname{Ei}(x)$ - интегральная показательная функция [3, с.56]. Доказано в [14, c. 23], что $F(x)$ при $x \rightarrow \infty$ имеет асимптотическое разложение:

$$
F(x) \sim \frac{1}{x}+\frac{1 !}{x^{2}}+\frac{2 !}{x^{3}}+\cdots+\frac{(k-1) !}{x^{k}}+\cdots,
$$

и при $x \rightarrow \infty$ для $k=1,2, \ldots$ справедлива формула

$$
F(x)=\frac{1}{x}+\frac{1 !}{x^{2}}+\frac{2 !}{x^{3}}+\cdots+\frac{(k-1) !}{x^{k}}+O\left(\frac{1}{x^{k+1}}\right) .
$$

Разложение (1.51) совпадает с рядом в (1.50). При $k=1$ из (1.52) получим

$$
F(x)=\frac{1}{x}+O\left(\frac{1}{x^{2}}\right), \quad x \rightarrow \infty .
$$

Из (1.50)-(1.53) будем иметь неравенство

$$
\left|R_{1}(t, \varepsilon)\right| \leqslant \frac{1}{x}+O\left(\frac{1}{x^{2}}\right), \quad x \rightarrow \infty .
$$

Из соотношения (1.53) следует

$$
F(x)=\frac{1}{x}+O\left(\frac{1}{x^{2}}\right)=\frac{1}{x}(1+o(1))=O\left(\frac{1}{x}\right), \quad x \rightarrow \infty,
$$

а это означает, что

$$
F(x) \leqslant A\left(\frac{1}{x}\right), \quad 1<a<x<\infty,
$$

где $A, a$ - постоянные.

Из (1.53)-(1.55) следует неравенство

$$
\left|R_{1}(t, \varepsilon)\right| \leqslant A\left(\frac{1}{x}\right)=4 A|\varepsilon|=A_{1}|\varepsilon|, \quad 0<|\varepsilon|<a_{1},
$$


что и доказывает соотношение

$$
\left.R_{1}(t, \varepsilon)=O(\varepsilon) \quad \text { при } \varepsilon \rightarrow 0 \text { (по } R\right) \text { в } G_{1}(\varepsilon) .
$$

Используя (1.35), запишем асимптотический ряд для $R_{2}^{(s)}(t, \varepsilon), s=1,2$, не выписывая индекс:

$$
R_{2}(t, \varepsilon)=R_{1}(t, \varepsilon)-\varepsilon u_{1}(t)=\sum_{k=2}^{\infty} \varepsilon^{k} u_{k}(t)
$$

Из (1.57) согласно неравенствам (1.36) аналогично (1.49) получим

$$
\left|R_{2}(t, \varepsilon)\right| \leqslant \frac{1 !}{x^{2}}+\frac{2 !}{x^{3}}+\cdots+\frac{(k-1) !}{x^{k}}+\cdots .
$$

Из разложения (1.51) следует, что в (1.58) ряд представляет асимптотическое разложение функции $F(x)-1 / x$ при $x \rightarrow \infty$, для которой по (1.52) при $k=2$ справедлива формула

$$
F(x)-\frac{1}{x}=\frac{1}{x^{2}}+O\left(\frac{1}{x^{3}}\right)=O\left(\frac{1}{x^{2}}\right), \quad x \rightarrow \infty .
$$

По формуле (1.59) из соотношения (1.58) получаем неравенство

$$
\left|R_{2}(t, \varepsilon)\right| \leqslant \frac{1}{x^{2}}+O\left(\frac{1}{x^{3}}\right), \quad x \rightarrow \infty .
$$

Далее, проведя выкладки, аналогичные (1.54)-(1.56), получим

$$
R_{2}(t, \varepsilon)=O\left(\varepsilon^{2}\right) \quad \text { при } \varepsilon \rightarrow 0(\text { по } R) \text { в } G_{1}(\varepsilon) .
$$

Проводя аналогичные доказательства для любого $n=2,3, \ldots$, при $s=1,2$ получим

$$
R_{n}(t, \varepsilon)=R_{1}(t, \varepsilon)-\sum_{k=1}^{n-1} \varepsilon^{k} u_{k}(t)=O\left(\varepsilon^{n}\right) \quad \text { при } \varepsilon \rightarrow 0(\text { по } R) \text { в } G_{1}(\varepsilon) .
$$

Из соотношений $(1.56),(1.61)$ следует, что ряды (1.36) при $s=1,2$ являются асимптотическими разложениями функций $R_{1}^{(s)}(t, \varepsilon)$ при $\varepsilon \rightarrow 0$ (по $R$ ) для $|\varepsilon|<1 / 4$ равномерно по $t$ при $|t| \leqslant 1$.

Пусть теперь $|t|>1$. Из формулы (1.36) следует, что при $s=1,2$ неравенство (1.49) примет вид

$$
R_{1}(t, \varepsilon) \leqslant \sum_{k=1}^{\infty}\left(4|t|^{3}|\varepsilon|\right)^{k}(k-1) !
$$

$\mathrm{B}(1.62)$ обозначим $4|t|^{3}|\varepsilon|=1 / x$ и получим формулу (1.50), в которой для любого фиксированного значения $t,|t|>1$, имеем $x \rightarrow \infty$ при $|\varepsilon| \rightarrow 0$. Далее доказательство аналогично (1.51)-(1.61). В результате при $n=1,2, \ldots$ получим оценки

$$
R_{n}(t, \varepsilon)=R_{1}(t, \varepsilon)-\sum_{k=1}^{n-1} \varepsilon^{k} u_{k}(t)=O\left(\varepsilon^{n}\right)
$$

при $\varepsilon \rightarrow 0$ (по $R$ ) в области $|\varepsilon|<1 /\left(4|t|^{3}\right)$, справедливые для любого фиксированного $t,|t|>1$. Соотношения (1.63) доказывают утверждение 2) при $|t|>1$. Лемма доказана. 
ТЕорема 1.2. В формуле (1.16) для фундаменталъной системы решений уравнения (1.1), записанной в виде

$$
w_{s}(z, \varepsilon)=\exp \left\{\frac{1}{\varepsilon} \int_{z_{0}}^{z} \lambda_{s}(\xi) d \xi\right\} D^{(s)}(z, \varepsilon),
$$

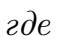

$$
D^{(s)}(z, \varepsilon)=\sum_{k=0}^{\infty} \varepsilon^{k} D_{k}^{(s)}(z), \quad D_{k}^{(2)}(z)=(-1)^{k} D_{k}^{(1)}(z), \quad s=1,2,
$$

ряды являются асимптотическими разложениями функиий $D^{(s)}(z, \varepsilon)$ при $\varepsilon \rightarrow 0($ по $R)$ в области $|\varepsilon|<1 / 4$, равномерными по z в области, определяемой неравенством $|t| \leqslant 1$, и неравномерными по $z$ для любого фиксированного $z \neq \pm 1$ в области $|t|>1$ при $|\varepsilon|<1 /\left(4|t|^{3}\right)$, где $t=1 / \sqrt{1-z^{2}}$.

$B$ плоскости $\overline{\mathbb{C}}(z)$ область равномерной сходимости ряда из (1.64) расположена на лемнискате $\rho=\sqrt{2 \cos (2 \varphi)}$ и вне ее, область неравномерной сходимости - внутри лемнискаты при $z \neq \pm 1$ (рис. 1, 2).

ДокАЗАТЕльство. В формуле (1.64) при $s=1,2$ (индекс не выписываем) согласно (1.28) имеем $D_{k}(z)=\sqrt{t} u_{k}(t), u_{0}(t)=1, k=0,1, \ldots$, и используем заключения леммы 1.1. В силу (1.34), (1.35), (1.61) справедливы соотношения

$$
\begin{gathered}
D(z, \varepsilon)=\sqrt{t}+\sqrt{t} R_{1}(t, \varepsilon)=\sqrt{t}+\sqrt{t} O(\varepsilon)=\sqrt{t}(1+o(1))=O(1), \\
D(z, \varepsilon)-\sum_{k=0}^{n-1} \varepsilon^{k} D_{k}(z)=\sqrt{t} R_{n}(t, \varepsilon)=\sqrt{t} O\left(\varepsilon^{n}\right)=O\left(\varepsilon^{n}\right) .
\end{gathered}
$$

Формулы (1.65) справедливы по лемме 1.1 при $\varepsilon \rightarrow 0$ (по $R$ ) равномерно по $t$ при $|t| \leqslant 1$ в области $|\varepsilon|<1 / 4$ и неравномерно по $t$ при $|t|>1$ в области $|\varepsilon|<$ $1 /\left(4|t|^{3}\right)$. Отсюда, учитывая формулу $t=1 / \sqrt{1-z^{2}}$, получаем доказательство теоремы.

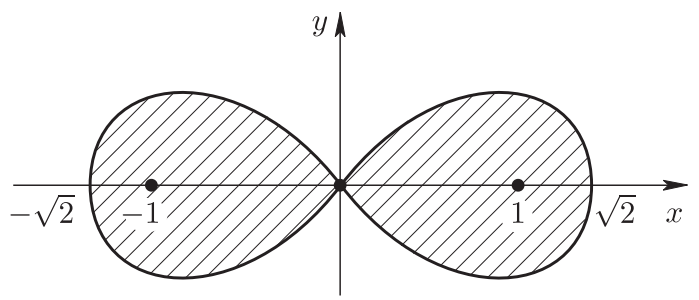

Рис. 1. Линия уровня (лемниската) функции $\Phi=|t(z)|$ при $\Phi=1$ в плоскости $z=x+i y$

В плоскости $\overline{\mathbb{C}}(z)$ линия $\left|1 / \sqrt{1-z^{2}}\right|=1$ в полярных координатах есть лемниската $\rho=\sqrt{2 \cos (2 \varphi)}$; для точек $z$ вне лемнискаты $|t|<1$, внутри нее $|t|>1$, за исключением точек $z= \pm 1$. Теорема доказана.

На рис. 1 область неравномерной сходимости рядов из (1.64) в области $G^{*}(z)$ заштрихована. На рис. 2 график рельефа функции $t(z)$ изображен при $z=x$. 


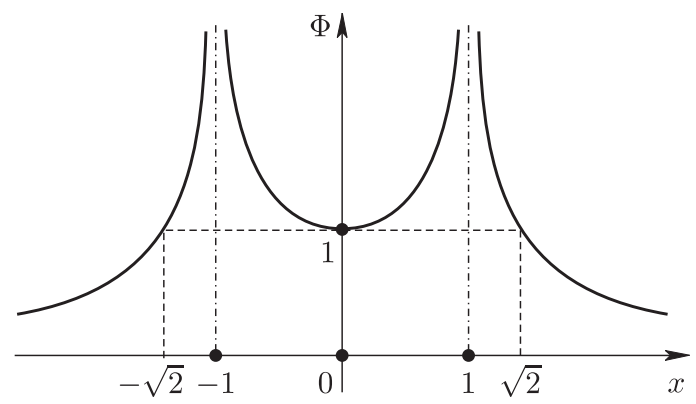

Рис. 2. Функция $\Phi=|t(z)|$ на оси $x$

СлЕДСТВИЕ 1.1. Формулъ (1.64) для ФСР уравнения (1.1) в области неравномерной сходимости рядов по $x$ для $\varepsilon=1 / \nu, \nu>0$, при $0<x<1$, $x=1 / \operatorname{ch} \alpha=\operatorname{sech} \alpha, \alpha>0$, совпадают с формулами асимптотических разложений Дебая ФСР уравнения (0.1) для функиий Бесселя $J_{\nu}(\nu \operatorname{sech} \alpha)$, $Y_{\nu}(\nu \operatorname{sech} \alpha)$ при $\nu \rightarrow \infty$ в изложении Ф. Ольвера [3, с. 187].

ДокАЗАТЕЛЬство. Запишем по теореме 1.2 при $D_{k}(z)=\sqrt{t} u_{k}(t)$ согласно (1.28) формулу (1.64) при $s=1, z=x, z_{0}=1, \varepsilon=1 / \nu$ в виде

$$
w_{1}\left(x, \frac{1}{\nu}\right)=\exp \left\{\nu \int_{1}^{x} \frac{\sqrt{1-\xi^{2}}}{\xi} d \xi\right\} \sqrt{t}\left(1+\sum_{k=1}^{\infty} \frac{u_{k}(t)}{\nu^{k}}\right) .
$$

В соотношении (1.66) произведем замену $x=1 / \operatorname{ch} \alpha=\operatorname{sech} \alpha$, где $0<\alpha<$ $+\infty, 0<\operatorname{sech} \alpha<1, t=1 / \sqrt{1-x^{2}}=\operatorname{cth} \alpha=1 /$ th $\alpha$, интеграл вычислим по формуле (1.5):

$$
\int_{1}^{x} \frac{\sqrt{1-\xi^{2}}}{\xi} d \xi=\sqrt{1-x^{2}}-\ln \left(\frac{1+\sqrt{1-x^{2}}}{x}\right)=\operatorname{th} \alpha-\alpha<0, \quad \alpha \neq 0,
$$

введем нормирующий множитель $1 / \sqrt{2 \pi \nu}$ и получим

$$
w_{1}\left(x, \frac{1}{\nu}\right)=\frac{\exp \{\nu(\operatorname{th} \alpha-\alpha)\}}{\sqrt{2 \pi \nu \operatorname{th} \alpha}}\left(1+\sum_{k=1}^{\infty} \frac{u_{k}(\operatorname{cth} \alpha)}{\nu^{k}}\right)=J_{\nu}(\nu x)
$$

при $\nu \rightarrow \infty$ для $\nu>4(\operatorname{cth} \alpha)^{3}$ и для любого фиксированного $x \in(0,1)$.

Формула (1.67) совпадает с формулой (9.3.7) из [3, с. 187] - формулой асимптотического разложения функции $J_{\nu}(\nu \operatorname{sech} \alpha)$.

При $s=2$ в формулу (1.66) введем нормирующий множитель $-\sqrt{2} / \sqrt{\pi \nu}$ и получим

$$
w_{2}\left(x, \frac{1}{\nu}\right)=-\frac{\sqrt{2} \exp \{\nu(\alpha-\operatorname{th} \alpha)\}}{\sqrt{\pi \nu \operatorname{th} \alpha}}\left(1+\sum_{k=1}^{\infty}(-1)^{k} \frac{u_{k}(\operatorname{cth} \alpha)}{\nu^{k}}\right)=Y_{\nu}(\nu x) .
$$

Формула (1.68) совпадает с формулой $(9.3 .8)$ из [3, с. 187] для $Y_{\nu}(\nu \operatorname{sech} \alpha)$. Формулы (9.3.7), (9.3.8) из [3] справедливы, если $\alpha$ фиксированно, при большом $\nu>0$, что согласуется с теоремой 1.2 для (1.67), (1.68), так как интервал 
$0<x<1$ находится в области неравномерной асимптотической сходимости рядов в $(1.66)$ при $\varepsilon \rightarrow 0(\nu \rightarrow \infty)$.

В [3, с. 187] коэффициенты рядов $u_{k}(t)$ определяются по формуле (9.3.10), совпадающей с (1.32), приведены значения $u_{k}(t), k=1,2,3,4$.

1.4. Исследование ФСР уравнения (1.1) по формуле (1.64) на действительной оси при $x>0$ и $\varepsilon>0$. Запишем уравнение (1.1) и формулу (1.64) для ФСР при $z=x, x_{0}=1$ в виде

$$
\begin{gathered}
L_{\varepsilon} u=\varepsilon^{2} x^{2} u^{\prime \prime}+\varepsilon^{2} x u^{\prime}-\left(1-x^{2}\right) u=0, \\
u_{s}(x, \varepsilon)=\exp \left\{\frac{(-1)^{s-1}}{\varepsilon} \int_{1}^{x} \frac{\sqrt{1-\xi^{2}}}{\xi} d \xi\right\} D^{(s)}(x, \varepsilon), \quad s=1,2,
\end{gathered}
$$

где

$$
D^{(s)}(x, \varepsilon)=\sum_{k=0}^{\infty} \varepsilon^{k} D_{k}^{(s)}(x), \quad D_{k}^{(2)}(x)=(-1)^{k} D_{k}^{(1)}(x) .
$$

Из теоремы 1.2 следует, что ряды в (1.69) являются асимптотическими разложениями функций $D^{(s)}(x, \varepsilon)$ на интервале $(0,+\infty)$, за исключением точки $x=1$, которая, как известно для уравнения Бесселя, является точкой перехода $[15$, пп. 4.5,4.8], поскольку при переходе через $x=1$ меняется тип поведения решения. В методе регуляризации для построения ФСР уравнения Бесселя (1.1) при $\varepsilon \rightarrow 0$ точка $x=1$ проявилась как особая точка функций спектра оператора Бесселя $\lambda_{s}(x), s=1,2$.

Будем исследовать ФСР на интервалах $(0,1)$ и $(1,+\infty)$, так как $\lambda_{s}(x)$ на $(0,1)$ имеет действительные значения, а на $(1,+\infty)$ - мнимые значения.

ТЕОРема 1.3. Формуль (1.69) являются регуляризованными асимптотическими разложениями при $\varepsilon \rightarrow+0$ для функций $u_{s}(x, \varepsilon), s=1,2$, неравномернъьми по $x \in(0,1)$ при $|\varepsilon|<1 /\left(4|t|^{3}\right)$, где $t=1 / \sqrt{1-x^{2}}$.

ДокАЗАТЕЛЬство. Из теоремы 1.2 следует, что интервал $(0,1)$ находится в области асимптотической сходимости ряда (1.69) при $\varepsilon \rightarrow+0$, следовательно, $D^{(s)}(x, \varepsilon)=O(1), \varepsilon \rightarrow+0$, а из характера поведения ФСР уравнения Бесселя в окрестности точки $x=0$ на действительной оси следует, что если одно решение ФСР ограничено, то другое не ограничено. В формуле (1.69) для $u_{1}(x, \varepsilon)$ экспоненциальный множитель при $x \in(0,1)$ для $\varepsilon>0$ ограничен, решение $u_{1}(x, \varepsilon)$ ограничено, следовательно, для $u_{1}(x, \varepsilon)$ формула (1.69) является асимптотическим разложением при $\varepsilon \rightarrow+0$. Для функции $u_{2}(x, \varepsilon)$ экспоненциальный множитель при $\varepsilon>0$ не ограничен на $(0,1)$, но так как функция $u_{2}(x, \varepsilon)$ не ограничена, то формулу (1.69) также принято называть ее асимптотическим разложением при $\varepsilon \rightarrow+0$, как это было проиллюстрировано в следствии 1.1 для функций $Y_{\nu}(\nu x)$.

ТЕОрема 1.4. Формула (1.69) при $x \in(1,+\infty)$ является регуляризованным асимптотическим разложением ФСР при $\varepsilon \rightarrow+0$ на $(1,+\infty)$, неравномерным на интервале $(1, \sqrt{2})$ и равномерным на $[\sqrt{2},+\infty)$, записанным в комплексном виде, из которого следует запись ФСР в действительном виде:

$$
\begin{aligned}
& u_{1}(x, \varepsilon)=A^{(1)}(x, \varepsilon) \cos \varphi(x, \varepsilon)-B^{(1)}(x, \varepsilon) \sin \varphi(x, \varepsilon), \\
& u_{2}(x, \varepsilon)=-A^{(2)}(x, \varepsilon) \sin \varphi(x, \varepsilon)+B^{(2)}(x, \varepsilon) \cos \varphi(x, \varepsilon),
\end{aligned}
$$


əдe

$$
\varphi(x, \varepsilon)=\frac{1}{\varepsilon} \int_{1}^{x} \frac{\sqrt{\xi^{2}-1}}{\xi} d \xi, \quad D^{(s)}(x, \varepsilon)=A^{(s)}(x, \varepsilon)+i B^{(s)}(x, \varepsilon), \quad s=1,2 .
$$

ДокАЗАТЕЛЬСтво следует из теоремы 1.2 при $z=x$ и формул (1.28).

\section{$\S 2$. Регуляризованное равномерное асимптотическое представление $Ф С Р$ уравнения (1.1) в окрестности регулярной особой точки}

По теореме 1.1 формальная регуляризованная ФСР записывается в виде (1.23). Рассмотрим ее при $z \in G=\{z:|z|<1\}$, принимая $t_{0}=1$ (что соответствует $z=0)$ в формуле (1.25), которая при $s=1$ имеет вид

$\hat{u}_{0}(t)=1, \quad \hat{u}_{k+1}(t)=\frac{1}{2} t^{2}\left(1-t^{2}\right) \hat{u}_{k}^{\prime}(t)+\frac{1}{8} \int_{1}^{t}\left(1-5 \zeta^{2}\right) \hat{u}_{k}(\zeta) d \zeta, \quad k=0,1, \ldots$,

где $\hat{u}_{k}(t)$ - многочлены по $t$ степени $3 k, \hat{u}_{k}(1)=0$. Первые $\hat{u}_{k}(t), k=1,2$, имеют следующий вид:

$$
\begin{gathered}
\hat{u}_{1}(t)=\frac{1}{8} t-\frac{5}{24} t^{3}+\frac{1}{12} \\
\hat{u}_{2}(t)=\frac{1}{96} t+\frac{9}{128} t^{2}-\frac{5}{288} t^{3}-\frac{77}{192} t^{4}+\frac{385}{1152} t^{6}+\frac{1}{288}, \\
\hat{u}_{k}^{(2)}(t)=(-1)^{k} \hat{u}_{k}(t) .
\end{gathered}
$$

Для доказательства асимптотической сходимости рядов при $\varepsilon \rightarrow 0$ из (1.23), (2.1) в области $G(z)$ согласно (1.28) запишем $\widehat{D}_{k}(z)=\sqrt{t} \hat{u}_{k}(t)$, где $\widehat{D}_{k}(z)$ - аналитические функции в $G(z), \widehat{D}_{0}(0)=1, \widehat{D}_{k}(0)=0, k=1,2, \ldots$ Формула $(1.23)$ в области $G(z)$ примет вид

$$
\widehat{w}_{s}(z, \varepsilon)=\exp \left\{\frac{1}{\varepsilon} \int_{z_{0}}^{z} \lambda_{s}(\xi) d \xi\right\} \widehat{D}^{(s)}(z, \varepsilon), \quad s=1,2,
$$

где

$$
\widehat{D}^{(s)}(z, \varepsilon)=\sum_{k=0}^{\infty} \varepsilon^{k} \widehat{D}_{k}^{(s)}(z), \quad \widehat{D}_{k}^{(2)}(z)=(-1)^{k} \widehat{D}_{k}^{(1)}(z) .
$$

ТЕОРема 2.1. В формуле (2.2) для ФСР ряди являются асимптотическими разложениями функиий $\widehat{D}^{(s)}(z, \varepsilon)$ в области $G(z)=\{z: 0 \leqslant|z|<1\}$, равномерными при $0 \leqslant|z| \leqslant R_{1}<1$ и при $\varepsilon \rightarrow 0$ для $s=1$ в области $\Omega_{1}(\varepsilon)=\{\varepsilon=$ $\left.R e^{i \varphi}, 0 \leqslant|\varphi|<\pi-\delta\right\}, \partial л я s=2$ в области $\Omega_{2}(\varepsilon)=\left\{\varepsilon=\operatorname{Re}^{i \varphi}, \delta<|\varphi| \leqslant \pi\right\}$, $0<R<\infty, 0<\delta<\pi / 2$. В области $\Omega=\Omega_{1} \cap \Omega_{2}$ эти разложения при $\varepsilon \rightarrow 0$ справедливы для двух решений $w_{s}(z, \varepsilon), s=1,2$.

ДокАЗАТЕЛьство. Оценим остаток ряда в (2.2) при любом $n=0,1, \ldots$ (индекс $s$ не выписываем):

$$
\begin{gathered}
R_{n}(z, \varepsilon)=\widehat{D}(z, \varepsilon)-\sum_{k=0}^{n} \varepsilon^{k} \widehat{D}_{k}(z)=\varepsilon^{n+1} V_{n}(z, \varepsilon), \\
V_{n}(z, \varepsilon)=\sum_{k=n+1}^{\infty} \varepsilon^{k} \widehat{D}_{k}(z), \quad V_{n}(0, \varepsilon)=0
\end{gathered}
$$


так как $\widehat{D}_{k}(0)=\hat{u}_{k}(1)=0, k=1,2, \ldots$. Определим уравнение для функции $V_{n}(z, \varepsilon)$, используя расширенную функцию из (1.14):

$$
\widetilde{w}(z, \tau, \varepsilon)=e^{\tau} \sum_{k=0}^{\infty} \varepsilon^{k} \widehat{D}_{k}(z)=e^{\tau} \widehat{D}(z, \varepsilon) .
$$

Она удовлетворяет уравнению (1.6), из которого при (2.5), (1.2) получим

$$
L_{\varepsilon} \widetilde{w}(z, \tau, \varepsilon)=\left[\varepsilon\left(z \widehat{D}_{z z}^{\prime \prime}(z, \varepsilon)+\widehat{D}_{z}^{\prime}(z, \varepsilon)\right)+2 z \lambda(z) \widehat{D}_{z}^{\prime}(z, \varepsilon)+(z \lambda(z))^{\prime} \widehat{D}(z, \varepsilon)\right] e^{\tau}=0 .
$$

Из (2.6) следует уравнение для $\widehat{D}(z, \varepsilon)$ (аргументы далее не выписываем):

$$
\tilde{L} \widehat{D} \equiv \varepsilon \tilde{L}_{2} \widehat{D}+\tilde{L}_{1} \widehat{D}=0
$$

Операторы $\tilde{L}_{1}, \tilde{L}_{2}$ определены в (1.12). Применим оператор $\tilde{L}$ ко второму равенству в (2.3):

$$
\tilde{L} \widehat{D}-\sum_{k=0}^{n} \varepsilon^{k} \tilde{L} \widehat{D}_{k}=\varepsilon^{n+1} \tilde{L} V_{n} .
$$

По формуле (2.7) из соотношения (2.8) получим

$$
\begin{aligned}
\varepsilon^{n+1} \tilde{L} V_{n} & =-\sum_{k=0}^{n} \varepsilon^{k} \tilde{L} \widehat{D}_{k}=-\sum_{k=0}^{n} \varepsilon^{k}\left(\varepsilon \tilde{L}_{2} \widehat{D}_{k}+\tilde{L}_{1} \widehat{D}_{k}\right) \\
& =-\tilde{L}_{1} \widehat{D}_{0}-\sum_{k=1}^{n} \varepsilon^{k}\left(\tilde{L}_{2} \widehat{D}_{k-1}+\tilde{L}_{1} \widehat{D}_{k}\right)-\varepsilon^{n+1} \tilde{L}_{2} \widehat{D}_{n}
\end{aligned}
$$

Из соотношения (1.13) имеем

$$
\tilde{L}_{1} \widehat{D}_{0}=0, \quad \tilde{L}_{2} \widehat{D}_{k-1}+\tilde{L}_{1} \widehat{D}_{k}=0 .
$$

Из (2.9) получаем уравнение для функции $V_{n}(z, \varepsilon): \tilde{L} V_{n}(z, \varepsilon)=-L_{2} \widehat{D}_{n}(z)$, которое в развернутом виде согласно (2.6), (2.7) будет следующим:

$$
\varepsilon z\left(V_{n}(z, \varepsilon)\right)_{z^{2}}^{\prime \prime}+(2 z \lambda(z)+\varepsilon)\left(V_{n}(z, \varepsilon)\right)_{z}^{\prime}+(z \lambda(z))^{\prime} V_{n}(z, \varepsilon)=-\left(z \widehat{D}_{n}^{\prime \prime}(z)+\widehat{D}_{n}^{\prime}(z)\right)
$$

Определим решение уравнения (2.11) в виде ряда:

$$
V_{n}(z, \varepsilon)=\sum_{m=1}^{\infty} \alpha_{n m}(\varepsilon) z^{m} .
$$

Разложим коэффициенты и правую часть в (2.11) по степеням $z$ :

$$
\begin{gathered}
\varepsilon z\left(V_{n}\right)_{z^{2}}^{\prime \prime}+\left(p_{0}(\varepsilon)+\sum_{k=1}^{\infty} p_{k} z^{k}\right)\left(V_{n}\right)_{z}^{\prime}+\left(\sum_{k=1}^{\infty} q_{k} z^{k}\right) V_{n}(z, \varepsilon)=\sum_{k=0}^{\infty} b_{n k} z^{k}, \\
p_{0}(\varepsilon)= \begin{cases}2+\varepsilon & \text { при } \lambda(z)=\lambda_{1}(z), \\
-2+\varepsilon & \text { при } \lambda(z)=\lambda_{2}(z) .\end{cases}
\end{gathered}
$$


Из (2.12)-(2.14) получаем рекуррентные формулы для коэффициентов $\alpha_{n m}(\varepsilon)$ функции $V_{n}(z, \varepsilon),(2.12)$ :

$$
\begin{gathered}
\alpha_{n 1}(\varepsilon)=\frac{b_{n 0}}{p_{0}(\varepsilon)}, \quad \alpha_{n 2}(\varepsilon)=\frac{b_{n 1}-p_{1} \alpha_{n 1}}{2\left(p_{0}(\varepsilon)+\varepsilon\right)}, \\
\alpha_{n m}(\varepsilon)=\frac{b_{n(m-1)}-\sum_{s=1}^{m-1}\left[s p_{m-s}+q_{m-1-s}\right] \alpha_{n s}(\varepsilon)}{m\left[p_{0}(\varepsilon)+(m-1) \varepsilon\right]}, \quad m=2,3, \ldots .
\end{gathered}
$$

Оценим $\left|\alpha_{n m}\right|$, предполагая $n$ фиксированным.

Удалим из $\varepsilon$-плоскости угловую область с вершиной в начале координат, симметричную относительно оси и содержащую точку $-p(0)$, с величиной угла $2 \delta$, где $0<\delta<\pi / 2$. В оставшейся части $\varepsilon$-плоскости $\Omega_{1}(\varepsilon)$ или $\Omega_{2}(\varepsilon)$ будет справедливо равенство

$$
\left|p_{0}(\varepsilon)+(m-1) \varepsilon\right|=\left|(m-1) \varepsilon-\left(-p_{0}(0)\right)\right|>\left|p_{0}(0)\right| \sin \delta=s, \quad m=1,2, \ldots .
$$

Для коэффициентов рядов в (2.13) справедливы следующие неравенства, равномерные по $z$ при $|z| \leqslant R_{1}<1$ :

$$
\left|p_{k}\right|<\frac{M_{1}}{R_{1}^{k}}, \quad\left|q_{k}\right|<\frac{M_{2}}{R_{1}^{k}}, \quad\left|b_{n k}\right|<\frac{M_{n 3}}{R_{1}^{k}},
$$

где $M_{i}$ - фиксированные постоянные для каждого решения $w_{s}(z, \varepsilon), s=1,2$.

Учитывая (2.16), (2.17), получим оценки коэффициентов $\alpha_{n m}(\varepsilon)$ в $(2.12)$ :

$$
\left|\alpha_{n 1}(\varepsilon)\right| \leqslant \frac{M_{n 3}}{s}<P_{n}
$$

(число $P_{n}$ будет определено ниже аналогично [4, п. 98]),

$$
\left|\alpha_{n 2}(\varepsilon)\right| \leqslant \frac{M_{n 3} / R_{1}+\left(M_{1} / R_{1}\right) P_{n}}{2 s}<\frac{M_{n}\left(P_{n}^{2}-1\right)}{R_{1}\left(P_{n}-1\right)}<\frac{P_{n}^{2}}{R_{1}},
$$

где $M_{n}=\frac{1}{s} \max \left\{M_{n 3}, M_{1}\right\}$. В (2.18) последнее неравенство эквивалентно очевидному неравенству $P_{n}^{2}\left[P_{n}-\left(1+M_{n}\right)\right]+M_{n}>0$, если выбрать $P_{n}>1+M_{n}$. В результате получим

$$
\left|\alpha_{n 2}(\varepsilon)\right|<\frac{P_{n}^{2}}{R_{1}} .
$$

Методом математической индукции доказывается, что для любых $\alpha_{n m}(\varepsilon)$ справедливо неравенство

$$
\left|\alpha_{n m}(\varepsilon)\right|<\frac{P_{n}^{m}}{R_{1}^{m-1}} .
$$

Ряд $R_{1} \sum_{k=0}^{\infty}\left(P_{n}^{k} / R_{1}^{k}\right) z^{k}$ сходится абсолютно в круге $|z|<R_{1} / P_{n}$. Следовательно, ряд (2.12) в силу неравенства (2.19) сходится в этом круге и определяет аналитическую функцию $V_{n+1}(z, \varepsilon)$, значит, и во всем круге $|z|<1$ при всех $\varepsilon \in \Omega_{1}(\varepsilon)$ или $\varepsilon \in \Omega_{2}(\varepsilon)$. Неравенство $\left|V_{n}(z, \varepsilon)\right|<C_{n}$ выполняется равномерно по $z$ при $|z| \leqslant \widetilde{R}<1$, постоянная $C_{n}$ не зависит от $\varepsilon \in \Omega_{s}(\varepsilon)$. Из формулы $(2.3)$ следует асимптотический характер рядов $(2.2)$ при $\varepsilon \rightarrow 0$ в $\Omega_{s}, s=1,2$, равномерный в области $\widetilde{G}(z)=\{z:|z| \leqslant \widetilde{R}<1\}$. В области $\Omega=\Omega_{1} \cap \Omega_{2}$ утверждения справедливы для решений $w_{s}(z, \varepsilon), s=1,2$. Теорема доказана. 
Теорема 2.2. В формуле (2.2) при $z=x, z_{0}=1$ для ФСР

$$
w_{s}(x, \varepsilon)=\exp \left\{\frac{(-1)^{s-1}}{\varepsilon} \int_{1}^{x} \frac{\sqrt{1-\xi^{2}}}{\xi} d \xi\right\} \widehat{D}^{(s)}(x, \varepsilon), \quad s=1,2,
$$

где

$$
\widehat{D}^{(s)}(x, \varepsilon)=\sum_{k=0}^{\infty} \varepsilon^{k} \widehat{D}_{k}^{(s)}(x), \quad \widehat{D}_{k}^{(2)}(x)=(-1)^{k} \widehat{D}_{k}^{(1)}(x),
$$

функции $\widehat{D}_{k}^{(s)}(x)$ являются аналитическими на $[0,1)$ при $s=1$ и удовлетворяют рекуррентным уравнениям системы (1.17):

$$
\begin{gathered}
2 \sqrt{1-x^{2}} \widehat{D}_{0}^{\prime}(x)-\frac{x \widehat{D}_{0}(x)}{\sqrt{1-x^{2}}}=0, \\
2 \sqrt{1-x^{2}} \widehat{D}_{k+1}^{\prime}(x)-\frac{x \widehat{D}_{k+1}(x)}{\sqrt{1-x^{2}}}=-\left(x \widehat{D}_{k}^{\prime \prime}(x)+\widehat{D}_{k}^{\prime}(x)\right), \quad k=0,1, \ldots,
\end{gathered}
$$

при условиях $\widehat{D}_{0}(0)=1, \widehat{D}_{k}(0)=0$.

Формулы (2.20) являются регуляризованными асимптотическими разложениями при $\varepsilon \rightarrow 0$, равномерными при $0 \leqslant x \leqslant a<1$ для решения $w_{1}(x, \varepsilon)$ в области $\operatorname{Re} \varepsilon \geqslant 0$ и для $w_{2}(x, \varepsilon)$ nри $\operatorname{Re} \varepsilon \leqslant 0$. На линиях Стокса [15, п. 3.5] $\varepsilon=i \eta(\eta \neq 0)$ оба решения $w_{s}(x, \varepsilon), s=1,2$, могут быть записаны в действительной форме по асимптотической последовательности $\left\{\eta^{k}\right\}, k=0,1, \ldots$, при $\eta \rightarrow 0$. Для $\eta>0$ имеем

$$
\begin{aligned}
& w_{1}(x, \eta)=A^{1}(x, \eta) \cos \varphi(x, \eta)+B^{1}(x, \eta) \sin \varphi(x, \eta), \\
& w_{2}(x, \eta)=-A^{1}(x, \eta) \sin \varphi(x, \eta)+B^{1}(x, \eta) \cos \varphi(x, \eta),
\end{aligned}
$$

где

$$
\varphi(x, \eta)=\left\{\frac{1}{\eta} \int_{1}^{x} \frac{\sqrt{1-\xi^{2}}}{\xi} d \xi\right\}, \quad \widehat{D}^{1}(x, \eta)=A^{1}(x, \eta)+i B^{1}(x, \eta) .
$$

ДокАЗАТЕЛЬство следует из формулы (1.17), теоремы 2.1, ограниченности экспоненциального множителя в указанных областях и свойств решений линейных дифференциальных уравнений.

ЗАмечание 2.1. Сравним формулу (2.2) с формулой Мейселя. В формуле $(2.2)$ используем соотношения $\widehat{D}_{k}^{(1)}(z)=\sqrt{t} \hat{u}_{k}(t)$, определяемые в $(2.1)$. Вычислим интеграл по формуле (1.5), введем нормирующий множитель, сделаем замену $\varepsilon=1 / \nu$. Формула (2.2) при $\nu \rightarrow \infty$ примет вид

$$
\widehat{w}_{1}(z, \varepsilon)=J_{\nu}(\nu z)=\frac{z^{\nu} \exp \left(\nu \sqrt{1-z^{2}}\right)}{\sqrt{2 \pi \nu}\left(1+\sqrt{1-z^{2}}\right) \sqrt[4]{1-z^{2}}} \sum_{k=0}^{\infty} \frac{\hat{u}_{k}(t)}{\nu^{k}} .
$$

В $\left[2\right.$, п. 8.11] приводится разложение Мейселя для $J_{\nu}(\nu z)$ при $\nu \rightarrow \infty$, в главной части совпадающее с формулой (2.23), но оно не является регуляризованным, так как регулярный ряд по степеням $1 / \nu$ стоит в показателе экспоненты. 
§ 3. Краевая задача для неоднородного и однородного уравнений Бесселя на отрезке $[0, a]$ при комплексном параметре

3.1. Постановка задачи и сведение ее к сингулярно возмущенной задаче. Для постановки краевой задачи и ее решения используем результаты предыдущих исследований регуляризованных асимптотических разложений $\Phi С Р$ для уравнения (1.1) при $x \in[0,1)$ и комплексном параметре $\varepsilon$ из $\S 2$.

Для уравнения Бесселя

$$
x^{2} w^{\prime \prime}+x w^{\prime}-\nu^{2}\left(1-x^{2}\right)=h(x)
$$

с комплексным параметром $\nu$ положим, что выполнено условие

$(*) \quad h(x)$ - аналитическая функция на $(-1,1)$ или $h(x) \equiv 0$.

Рассмотрим краевую задачу на $[0, a], 0<a<1$ с условиями

$$
w(x, \nu)=O(1), \quad x \rightarrow 0, \quad w(a, \nu)=w^{0}, \quad w^{0} \in \mathbb{R}^{1}, \quad w^{0} \neq 0 .
$$

Введем обозначение $\nu^{2}=1 / \varepsilon^{2}$ и сведем задачу (3.1), (3.2) к следующей сингулярно возмущенной задаче при $\varepsilon \rightarrow 0$ :

$$
\begin{gathered}
L_{\varepsilon} w \equiv \varepsilon^{2} x^{2} w^{\prime \prime}+\varepsilon^{2} x w^{\prime}-\left(1-x^{2}\right) w=\varepsilon^{2} h(x), \\
w(x, \varepsilon)=O(1), \quad x \rightarrow 0, \quad w(a, \varepsilon)=w^{0}, \quad w^{0} \neq 0 .
\end{gathered}
$$

Построим регуляризованные, асимптотические при $\varepsilon \rightarrow 0$, равномерные по $x$ решения краевой задачи (3.3), (3.4) в областях и на линиях Стокса в плоскости $\mathbb{C}(\varepsilon)$. Для решения задачи (3.3), (3.4) применим метод регуляризации [1].

3.2. Построение формальных регуляризованных решений задачи (3.3), (3.4). Вместо искомого решения $w(x, \varepsilon)$ введем расширенную функцию $\widehat{w}(x, \tau, \varepsilon)$, за дополнительную переменную $\tau$ примем регуляризирующую функцию

$$
\tau=-\frac{1}{\varepsilon} \int_{x}^{a} \lambda(\xi) d \xi \equiv \varphi(x, \varepsilon),
$$

где $\lambda(x)=\lambda_{1}(x)$ - корень характеристического уравнения (1.2). Потребуем, чтобы функция $\widehat{w}(x, \tau, \varepsilon)$ имела своим сужением при $\tau=\varphi(x, \varepsilon)$ искомое решение задачи (3.3), (3.4).

Из (3.3)-(3.5) получаем следующую краевую задачу для функции $\widehat{w}(x, \tau, \varepsilon)$ :

$$
\begin{gathered}
\varepsilon^{2} L_{2} \widehat{w}+\varepsilon L_{1} \widehat{w}+L_{0} \widehat{w}=\varepsilon^{2} h(x), \\
L_{0} w \equiv x^{2} \lambda^{2}(x) \frac{\partial^{2} w}{\partial \tau^{2}}-\left(1-x^{2}\right) w, \quad L_{1} w \equiv 2 x^{2} \lambda(x) \frac{\partial^{2} w}{\partial x \partial \tau}+x(x \lambda(x))^{\prime} \frac{\partial w}{\partial \tau}, \\
L_{2} w \equiv x^{2} \frac{\partial^{2} w}{\partial x^{2}}+x \frac{\partial w}{\partial x}
\end{gathered}
$$

с условиями

$$
\widehat{w}(x, \tau, \varepsilon)=O(1), \quad x \rightarrow 0, \quad \widehat{w}(a, 0, \varepsilon)=w^{0} .
$$

Задача (3.6), (3.7) регулярна по $\varepsilon$. Определим ее решение в виде ряда:

$$
\widehat{w}(x, \tau, \varepsilon)=\sum_{k=0}^{\infty} \varepsilon^{k} v_{k}(x, \tau) .
$$


Из уравнения (3.6) получаем уравнения для $v_{k}(x, \tau)$ :

$$
\begin{gathered}
L_{0} v_{0}(x, \tau)=0, \quad L_{0} v_{1}(x, \tau)=-L_{1} v_{0}(x, \tau), \\
L_{0} v_{2}(x, \tau)=-L_{1} v_{1}(x, \tau)-L_{2} v_{0}(x, \tau)+h(x), \\
L_{0} v_{k}(x, \tau)=-L_{1} v_{k-1}(x, \tau)-L_{2} v_{k-2}(x, \tau), \quad k=3,4, \ldots
\end{gathered}
$$

Краевые условия определяются из (3.7), (3.8):

$$
\begin{gathered}
\left|v_{k}(x, \tau)\right|<\widetilde{C}, \quad x \rightarrow 0, \quad k=0,1,2, \\
v_{k}(x, \tau) \rightarrow 0, \quad k=3,4, \ldots, \\
v_{0}(a, 0)=w^{0}, \quad v_{k}(a, 0)=0, \quad k=1,2, \ldots .
\end{gathered}
$$

Согласно идее метода регуляризации для решения задачи (3.9), (3.10) определим пространство безрезонансных решений $U$ следующим образом:

$$
U=\left\{v_{k}: v_{k}(x, \tau)=b_{k}(x) e^{\tau}+y_{k}(x)\right\} .
$$

Пространство $U$ двумерное, его базис состоит из элементов $v_{1}^{*}=e^{\tau}=\{1,0\}$, $v_{2}^{*}=1=\{0,1\}$.

В пространстве $U$ при каждом фиксированном $x$ определим скалярное произведение

$$
\left\langle v_{i}, v_{j}\right\rangle=b_{i}(x) b_{j}(x)+y_{i}(x) y_{j}(x) .
$$

Определим результаты действия операторов $L_{n}, n=0,1,2$, из (3.6) на функции $v_{k}(x, \tau) \in U, k=0,1, \ldots$ :

$$
\begin{aligned}
& L_{0} v_{k} \equiv\left[x^{2} \lambda^{2}(x)-\left(1-x^{2}\right)\right] b_{k}(x) e^{\tau}-\left(1-x^{2}\right) y_{k} \equiv-\left(1-x^{2}\right) y_{k}, \\
& L_{1} v_{k} \equiv x\left[2 x \lambda(x) b_{k}^{\prime}+(x \lambda(x))^{\prime} b_{k}(x)\right] e^{\tau} \equiv x e^{\tau} \tilde{L}_{1} b_{k}(x), \\
& L_{2} v_{k} \equiv x\left[x b_{k}^{\prime \prime}+b_{k}^{\prime}(x)\right] e^{\tau}+x^{2} y_{k}^{\prime \prime}+x y_{k}^{\prime} \equiv x e^{\tau} \tilde{L}_{2} b_{k}(x)+\tilde{L}_{3} y_{k}(x) .
\end{aligned}
$$

Операторы $\tilde{L}_{1}, \tilde{L}_{2}$ определены в формулах (1.12).

Из (3.9), (3.13) получаем следующие рекуррентные уравнения для определения функций $b_{k}(x), y_{k}(x)$ :

$$
\begin{gathered}
L_{0} v_{0}(x, \tau)=0, \quad L_{0} v_{1}(x, \tau)=-x e^{\tau} \tilde{L}_{1} b_{0}(x), \\
L_{0} v_{2}(x, \tau)=-x e^{\tau}\left[\tilde{L}_{1} b_{1}(x)+\tilde{L}_{2} b_{0}(x)\right]-\tilde{L}_{3} y_{0}(x)+h(x), \\
L_{0} v_{k}(x, \tau)=-x e^{\tau}\left[\tilde{L}_{1} b_{k-1}(x)+\tilde{L}_{2} b_{k-2}(x)\right]-\tilde{L}_{3} y_{k-2}(x), \quad k=3,4, \ldots .
\end{gathered}
$$

Краевые условия определим согласно (3.10), (3.11):

$$
\begin{gathered}
\left|b_{k}(0)\right|<\infty, \quad k=0,1, \ldots, \quad y_{k}(0)=0, \quad k=0,1,3, \ldots, \quad\left|y_{2}(0)\right|<\infty, \\
b_{0}(a)+y_{0}(a)=w^{0}, \quad b_{k}(a)+y_{k}(a)=0, \quad k=1,2, \ldots
\end{gathered}
$$

Запишем уравнения (3.14) в виде

$$
L_{0} v_{i}(x, \tau)=f_{i}(x, \tau), \quad f_{i}(x, \tau) \in U, \quad i=1,2, \ldots
$$

Пространство $U$ конечномерно, оператор $L_{0}$ нормально разрешим, и по теории метода регуляризации $[1$, с.45] следует, что для разрешимости системы (3.17) в $U$ необходимо и достаточно, чтобы $\left\langle f_{i}, \operatorname{ker} L_{0}^{*}\right\rangle \equiv 0$ (тождественно 
по $x)$. В пространстве $U$ оператор $L_{0}$ самосопряженный, базисом ядра в $U$ является элемент $v_{1}^{*}=e^{\tau}=\{1,0\}$, условия ортогональности принимают вид

$$
\left\langle f_{i}, v_{1}^{*}\right\rangle=0, \quad i=0,1, \ldots
$$

При условиях (3.18) краевые задачи (3.13)-(3.16) однозначно разрешимы. Поскольку $L_{0} v_{0} \equiv-\left(1-x^{2}\right) y_{0}$, то из первого уравнения (3.14) находим

$$
y_{0}(x) \equiv 0 \text {. }
$$

Второе уравнение из (3.14) будет иметь вид

$$
L_{0} v_{1}(x, t)=-x\left[2 x \lambda(x) b_{0}^{\prime}(x)+(x \lambda(x))^{\prime} b_{0}(x)\right] e^{\tau} .
$$

Правая часть принадлежит пространству $U$. Применяя к ней условие ортогональности $(3.18)$, для функции $b_{0}(x)$ получим уравнение

$$
\tilde{L}_{1} b_{0}(x) \equiv 2 \sqrt{1-x^{2}} b_{0}^{\prime}(x)-\frac{x}{\sqrt{1-x^{2}}} b_{0}(x)=0
$$

с начальным условием $b_{0}(a)=w^{0}$.

Из (3.20) имеем

$$
b_{0}(x)=\frac{\sqrt[4]{1-a^{2}} w^{0}}{\sqrt[4]{1-x^{2}}}, \quad b_{0}(0)=\sqrt[4]{1-a^{2}} w^{0}=D
$$

При этом получаем уравнение $L_{0} v_{1}(x, \tau)=0$, эквивалентное $-\left(1-x^{2}\right) y_{1}(x)=0$, из которого определим

$$
y_{1}(x) \equiv 0
$$

Третье уравнение в (3.14) будет следующим:

$$
\left(1-x^{2}\right) y_{2}(x)=x\left[\tilde{L}_{1} b_{1}+\tilde{L}_{2} b_{0}(x)\right] e^{\tau}-h(x) .
$$

Из условия ортогональности (3.18) получаем линейное неоднородное уравнение первого порядка для определения функции $b_{1}(x)$ :

$$
\tilde{L}_{1} b_{1}(x)=-\left(x b_{0}^{\prime \prime}+b_{0}^{\prime}(x)\right), \quad b_{1}(a)=0 .
$$

Начальное условие получили из (3.16), (3.22).

Общее решение уравнения (3.24) запишем в виде

$$
b_{1}(x)=\frac{C}{\sqrt[4]{1-x^{2}}}+D_{1}^{(1)}(x)
$$

где $D_{1}^{(1)}(x)$ - частное решение уравнения (3.24), удовлетворяющее условию $D_{1}^{(1)}(0)=0$. Из начального условия определим $C$, и в результате функция определится формулой

$$
b_{1}(x)=-\sqrt[4]{\frac{1-a^{2}}{1-x^{2}}} D_{1}^{(1)}(a)+D_{1}^{(1)}(x), \quad\left|b_{1}(0)\right|=\left|-\sqrt{1-a^{2}} D_{1}^{(1)}(a)\right|<\infty .
$$


Теперь уравнение (3.23) примет вид $\left(1-x^{2}\right) y_{2}(x)=-h(x)$, из него определим функцию

$$
y_{2}(x)=-\frac{h(x)}{1-x^{2}} .
$$

Методом математической индукции доказывается, что таким образом последовательно определяются функции $b_{k}(x)$ из рекуррентных задач

$$
\tilde{L}_{1} b_{k}(x)=-\tilde{L}_{2} b_{k-1}(x), \quad b_{k}(a)=-y_{k}(a)
$$

по следующим формулам:

$$
b_{k}(x)=-\sqrt[4]{\frac{1-a^{2}}{1-x^{2}}}\left(D_{k}^{(1)}(a)+y_{k}(a)\right)+D_{k}^{(1)}(x), \quad k=1,2, \ldots,
$$

где функции $D_{k}^{(1)}(x)$ - частные решения уравнения $(3.27)$ при $D_{k}^{(1)}(0)=0$. Функции $y_{k}(x)$ определяются далее из рекуррентных уравнений

$$
\left(1-x^{2}\right) y_{k}(x)=\tilde{L}_{3} y_{k-2}(x), \quad k=3,4, \ldots, \quad y_{k}(0)=0 .
$$

Все функции $b_{k}(x)$ и $y_{k}(x), k=0,1, \ldots$, являются аналитическими при $x \in$ $[0,1)$ и однозначно определены, откуда следует, что однозначно определяются функции

$$
v_{k}(x, t)=e^{t} b_{k}(x)+y_{k}(x)
$$

из пространства $U$.

Подставляем $v_{k}(x, t),(3.30)$, в ряд (3.8) и получаем решение задачи (3.9), (3.10) в виде регуляризованного ряда с коэффициентами из пространства $U$ :

$$
\widehat{w}(x, \tau, \varepsilon)=\sum_{k=0}^{\infty} \varepsilon^{k}\left(e^{\tau} b_{k}(x)+y_{k}(x)\right) .
$$

Проведем сужение этого решения на регуляризирующую функцию $\tau=\varphi(z, \varepsilon)$, в результате получим формальное регуляризованное решение при $h(x) \not \equiv 0$ краевой задачи (3.3), (3.4):

$$
w_{\mathrm{I}}(x, \varepsilon)=\exp \left(-\frac{1}{\varepsilon} \int_{x}^{a} \lambda_{1}(\xi) d \xi\right) \sum_{k=0}^{\infty} \varepsilon^{k} b_{k}(x)+\sum_{k=0}^{\infty} \varepsilon^{k} y_{k}(x) .
$$

Решение (3.31) уравнения (3.3) состоит из суммы двух рядов. Первый ряд является решением однородного уравнения. Второй ряд $\sum_{k=0}^{\infty} \varepsilon^{k} y_{k}(x)$, называемый основным, является формальным решением уравнения (3.3) при $h(x) \not \equiv 0$ и совпадает с рядом, который получается непосредственной подстановкой формального ряда по степеням $\varepsilon$ в уравнение (3.3).

3.3. Доказательство асимптотической сходимости основного ряда. Поскольку $y_{0}(x) \equiv 0, y_{2 n-1}(x) \equiv 0, n=1,2, \ldots$, то основной ряд будет иметь вид $\sum_{k=1}^{\infty} \varepsilon^{2 k} y_{2 k}(x)$.

ТЕОРема 3.1. Основной ряд $\sum_{k=0}^{\infty} \varepsilon^{k} y_{k}(x)$ в (3.31) является асимптотическим разложением аналитического по $x$ решения $w(x, \varepsilon)$ уравнения (3.3) при $h(x) \not \equiv 0$, равномерным по $x \in[0, a]$ при $\varepsilon \rightarrow 0$ в области

$$
\Omega=\left\{\varepsilon: \delta<|\arg \varepsilon|<\pi-\delta, 0<\delta<\frac{\pi}{2}\right\} .
$$


ДокАЗАТЕЛЬСтво. В уравнении (3.3) сделаем аналитическое продолжение коэффициентов и $h(x)$ в комплексную плоскость $z$. Рассмотрим уравнение (3.3) и его решение $w(z, \varepsilon)$ в области $\widetilde{G}=\{z:|z|<R, a<R<1\}$.

Пусть $w(z, \varepsilon)$ - точное решение уравнения (3.3), а $w_{2 n}(z, \varepsilon)$ - асимптотическое решение порядка $2 n$. Остаточный член обозначим

$$
\begin{gathered}
R_{2 n}(z, \varepsilon)=w(z, \varepsilon)-w_{2 n}(z, \varepsilon)=\varepsilon^{2(n+1)} V_{n}(z, \varepsilon), \\
V_{n}(z, \varepsilon)=\sum_{k=0}^{\infty} \varepsilon^{2 k} y_{2(n+1+k)}(z), \quad V_{n}(0, \varepsilon)=0, \quad n=1,2, \ldots
\end{gathered}
$$

Определим уравнение, которому удовлетворяет функция $V_{n}(z, \varepsilon)$, действуя оператором $L_{\varepsilon},(3.3)$, на первое равенство в (3.33):

$$
L_{\varepsilon} w-\sum_{k=1}^{n} \varepsilon^{2 k} L_{\varepsilon} y_{2 k}(z)=\varepsilon^{2(n+1)} L_{\varepsilon} V_{n}(z, \varepsilon) \text {. }
$$

Поскольку $L_{\varepsilon} w=\varepsilon^{2} h(z)$, то с учетом формул (3.26), (3.29) получим уравнение

$$
L_{\varepsilon} V_{n}(z, \varepsilon)=-\left(z^{2} y_{2 n}^{\prime \prime}(z)+z y_{2 n}^{\prime}(z)\right) .
$$

Будем определять решение уравнения (3.34) в виде ряда:

$$
V_{n}(z, \varepsilon)=\sum_{k=1}^{\infty} a_{n k}(\varepsilon) z^{k}
$$

Разложим правую часть уравнения (3.34) в ряд по степеням $z$ и запишем уравнение в развернутом виде:

$$
\varepsilon^{2} z^{2} V_{n}^{\prime \prime}+\varepsilon^{2} z V_{n}^{\prime}-\left(1-z^{2}\right) V_{n}=\sum_{k=1}^{\infty} b_{n k} z^{k},
$$

где $\left|b_{n k}\right|<M_{n} / R_{1}^{k}$ и $M_{n}$ - некоторая постоянная, $0<R_{1}<R$. Подставляя ряд (3.35) в уравнение (3.36), получим для коэффициентов $a_{n k}(\varepsilon)$ рекуррентные формулы

$$
a_{n 1}(\varepsilon)=\frac{b_{n 1}}{\varepsilon^{2}-1}, \quad a_{n 2}(\varepsilon)=\frac{b_{n 2}}{2^{2} \varepsilon^{2}-1}, \quad a_{n k}(\varepsilon)=\frac{b_{n k}-a_{n(k-2)}(\varepsilon)}{k^{2} \varepsilon^{2}-1}, \quad k \geqslant 3 .
$$

В области $\Omega$ для любого $\varepsilon$ справедливы неравенства

$$
|k \varepsilon-1|>\sin \delta=s, \quad|k \varepsilon+1|>\sin \delta=s .
$$

Учитывая (3.38), получим следующие оценки для $a_{n k}(\varepsilon)$ :

$$
\left|a_{n 1}(\varepsilon)\right|<\frac{M_{n}}{R_{1} s^{2}}<\frac{P_{n}}{R_{1}}
$$

(число $P_{n}$ будет определено ниже),

$$
\begin{gathered}
\left|a_{n 2}(\varepsilon)\right|<\frac{M_{n}}{R_{1}^{2} s^{2}}<\frac{P_{n}}{R_{1}^{2}}, \\
\left|a_{n 3}(\varepsilon)\right|=\left|\frac{b_{n 3}-a_{n 1}}{3^{2} \varepsilon^{2}-1}\right|<\frac{\frac{M_{n}}{R_{1}^{3}}+\frac{P_{n}}{R_{1}}}{s^{2}}<\frac{K_{n}\left(1+P_{n}\right)}{R_{1}^{3}}<\frac{K_{n}\left(P_{n}^{2}-1\right)}{R_{1}^{3}\left(P_{n}-1\right)}<\frac{P_{n}^{2}}{R_{1}^{3}},
\end{gathered}
$$

где $K_{n}=\frac{1}{s^{2}} \max \left\{M_{n}, R_{1}^{2}\right\}, P_{n}>1+K_{n}$ (см. (2.18)). 
Методом математической индукции доказывается, что

$$
\left|a_{n k}(\varepsilon)\right|<\frac{P_{n}^{k-1}}{R_{1}^{k}}, \quad k=2,3, \ldots .
$$

Из соотношений (3.40) следует, что ряд (3.35) сходится при $|z|<R_{1} / P_{n}$, и так как уравнение (3.34) не имеет других особых точек в области $\widetilde{G}$, кроме $z=0$, то при аналитическом продолжении сумма ряда не будет иметь особых точек в области $\widetilde{G}$, следовательно, ряд сходится при $|z|<R$, сумма ряда $V_{n}(z, \varepsilon)$ есть функция, аналитическая по $z$ в области $\widetilde{G}$ при любых $\varepsilon \in \Omega$. Функция $V_{n}(z, \varepsilon)$ как сумма степенного ряда (3.35) будет ограничена по модулю: $\left|V_{n}(z, \varepsilon)\right|<B_{n}-$ равномерно по $z$ при $|z| \leqslant a<R$, постоянная $B_{n}$ не зависит от $\varepsilon \in \Omega$. Таким образом, из (3.33) получаем

$$
\left|w(z, \varepsilon)-\sum_{k=0}^{n} \varepsilon^{2 k} y_{2 k}(z)\right| \leqslant|\varepsilon|^{2 n+2} B_{n} .
$$

Поскольку $y_{0}(z) \equiv 0, y_{2 k-1}(z) \equiv 0, k=1,2, \ldots$, то из (3.41) следует, что справедливы неравенства

$$
\left|w(z, \varepsilon)-\sum_{k=0}^{n} \varepsilon^{k} y_{k}(z)\right| \leqslant|\varepsilon|^{n+1} C_{n},
$$

равномерные по $z$ при $|z| \leqslant a, C_{n}$ не зависит от $\varepsilon \in \Omega$. Из (3.42) следует утверждение теоремы.

Теорема 3.2. Пусть выполнено условие (*); тогда краевая задача (3.3), (3.4) имеет равномерное по $x$ регуляризованное асимптотическое решение $w_{\mathrm{I}}(x, \varepsilon)$ nри $\lambda_{1}(x)$, выражаемое формулой (3.31) для $h(x) \not \equiv 0$ при $\varepsilon \rightarrow 0$ в области $E_{1}^{\delta}=\{\varepsilon: \delta<|\arg \varepsilon| \leqslant \pi / 2\}$ и для $h(x) \equiv 0$ в области $E_{1}=\{\varepsilon: \operatorname{Re} \varepsilon \geqslant 0\}$.

ДокАЗАТЕЛЬСтво. Подставим функции $b_{n}(x),(3.21),(3.28)$, в соотношение $(3.31)$ и получим решение $w_{\mathrm{I}}(x, \varepsilon)$ в виде

$$
\begin{aligned}
w_{\mathrm{I}}(x, \varepsilon)=\exp & \left(-\frac{1}{\varepsilon} \int_{x}^{a} \lambda_{1}(\xi) d \xi\right)\left(\frac{w^{0} \sqrt[4]{1-a^{2}}}{\sqrt[4]{1-x^{2}}}+\sum_{k=1}^{\infty} \varepsilon^{k}\left[D_{k}^{(1)}(x)\right.\right. \\
& \left.\left.-\sqrt[4]{\frac{1-a^{2}}{1-x^{2}}}\left(D_{k}^{(1)}(a)+y_{k}(a)\right)\right]\right)+\sum_{k=0}^{\infty} \varepsilon^{k} y_{k}(x), \quad D_{k}^{(1)}(0)=0 .
\end{aligned}
$$

В формуле (3.43) функции $b_{0}(x), D_{k}^{(1)}(x), k=1,2, \ldots$, как следует из формул (3.20), (3.27), удовлетворяют системе (2.21), следовательно, по теоремам $2.1,2.2,3.1$, свойствам асимптотических разложений и неравенству в $E_{1}$

$$
\left|\exp \left(-\frac{1}{\varepsilon} \int_{x}^{a} \lambda_{1}(\xi) d \xi\right)\right| \leqslant 1
$$

будет следовать, что формула (3.31) определяет равномерное по $x$ регуляризованное асимптотическое разложение решения $w_{\mathrm{I}}(x, \varepsilon)$ задачи $(3.3),(3.4)$ при $\varepsilon \rightarrow 0$ в указанных областях. Условия (3.4) выполнены. 
Все изложенное при решении краевой задачи $(3.3),(3.4)$ в области $E_{1}^{(\delta)}(\varepsilon)$ при $h(x) \not \equiv 0$ (в $E_{1}$ при $h(x) \equiv 0$ ) можно аналогично провести при $\lambda(x)=\lambda_{2}(x)$ и получить аналогичный результат для решения $w_{\mathrm{II}}(x, \varepsilon)$ в области $E_{2}^{(\delta)}(\varepsilon)=$ $\{\varepsilon: \pi / 2 \leqslant|\arg \varepsilon|<\pi-\delta\}$ при $h(x) \not \equiv 0$ и в области $E_{2}(\varepsilon)=\{\varepsilon: \pi / 2 \leqslant|\arg \varepsilon| \leqslant \pi\}$ для $h(x) \equiv 0$. В итоге получим формулу

$$
w_{\mathrm{II}}(x, \varepsilon)=\exp \left(-\frac{1}{\varepsilon} \int_{x}^{a} \lambda_{2}(\xi) d \xi\right) \sum_{n=0}^{\infty}(-1)^{n} \varepsilon^{n} b_{n}(x)+\sum_{n=0}^{\infty} \varepsilon^{n} y_{n}(x) .
$$

3.4. Решение задачи на линиях Стокса. В комплексной плоскости $\varepsilon=\gamma+i \eta$ на линиях $S_{k}, k=1,2\left(\varepsilon=(-1)^{k+1} i \eta, \eta \neq 0\right)$, будут справедливы формулы $(3.31)$ для $w_{\mathrm{I}}(x, \varepsilon)$ и $(3.44)$ для $w_{\mathrm{II}}(x, \varepsilon) ; S_{k}$ - линии Стокса в плоскости $\varepsilon\left[15\right.$, п. 3.5]. Уравнение (3.3) на $S_{1}$ будет иметь вид

$$
\eta^{2} x^{2} w^{\prime \prime}+\eta^{2} x w^{\prime}+\left(1-x^{2}\right) w=\eta^{2} h(x) .
$$

Решение задачи (3.45), (3.3), (3.4) можно записать в действительной форме:

$$
\begin{aligned}
w(x, \eta) & =\left(\cos \frac{1}{\eta} \int_{x}^{a} \lambda_{1}(\xi) d \xi\right) \sum_{n=0}^{\infty}(-1)^{n} \eta^{2 n} b_{2 n}(x) \\
+ & \left(\sin \frac{1}{\eta} \int_{x}^{a} \lambda_{1}(\xi) d \xi\right) \sum_{n=1}^{\infty}(-1)^{n-1} \eta^{2 n-1} b_{2 n-1}(x)+\sum_{n=1}^{\infty}(-1)^{n} \eta^{2 n} y_{2 n}(x) .
\end{aligned}
$$

Если перейти в формулах $(3.31),(3.44),(3.46)$ от $\varepsilon$ к $\nu$ по формуле $\varepsilon=1 / \nu$, то получим асимптотические решения задачи (3.1), (3.2) при $\nu \rightarrow \infty$.

\section{Список литературы}

1. С.А. Ломов, Введение в общую теорию сингулярных возмущений, Наука, М., 1981; англ. пер.: S. A. Lomov, Introduction to the general theory of singular perturbations, Transl. Math. Monogr., 112, Amer. Math. Soc., Providence, RI, 1992.

2. Г. Н. Ватсон, Теория бесселевых функиий, ИЛ, М., 1949; пер. с англ.: G. N. Watson, A treatise on the theory of Bessel functions, Cambridge Univ. Press, Cambridge; Macmillan, New York, 1944.

3. М. Абрамовиц, М. Стиган, Справочник по специальным функциям с формулами, графиками и математическими таблицами, Наука, М., 1979; пер. с англ.: M. Abramowitz, I. A. Stegun, Handbook of mathematical functions with formulas, graphs, and mathematical tables, U.S. Government Printing Office, Washington, 1964.

4. В.И. Смирнов, Курс высшей математики, т. III, ч. 2, Физматгиз, М., 1958; англ. пер.: V.I. Smirnov, A course of higher mathematics. Vol. III. Part two. Complex variables. Special functions, Transl. Math. Monogr., 60, Pergamon Press, Oxford-Edinburgh-New York; Addison-Wesley, Reading-London, 1964.

5. А. С. Юдина, "Асимптотические разложения цилиндрических функций от двух действительных переменных", ЖКМ и МФ, 1:6 (1961), 1099-1104; англ. пер.: A.S. Yudina, "Asymptotic expansions of cylindrical functions of two real variables", U.S.S.R. Comput. Math. and Math. Phys., 1:6 (1962), 1277-1283.

6. П. И. Кузнецов, А. С. Юдина, "Некоторые свойства нулей цилиндрических функций от двух действительных переменных", ЖВМ и МФ, 12:3 (1972), 739-746; англ. пер.: P. I. Kuznetsov, A. S. Yudina, "Some properties of the zeros of the cylinder functions of two real variables", U.S.S.R. Comput. Math. and Math. Phys., 12:3 (1972), 208-218. 
7. П. И. Кузнецов, А. С. Юдина, "Некоторые асимптотические разложения неполного интеграла вероятности", Теория вероятн. и ее примен., 18:2 (1973), 367-371; англ. пер.: P.I. Kuznetsov, A.S. Yudina, "Some asymptotic expansions for an incomplete probability integral", Theory Probab. Appl., 18 (1973), 355-359.

8. П. И. Кузнецов, А. С. Юдина, “Асимптотические решения вырожденного гипергеометрического уравнения", Дифферени. уравнения, 23:3 (1987), 428-434; англ. пер.: P. I. Kuznetsov, A. S. Yudina, "Asymptotic expansions of solutions of a confluent hypergeometric equation", Differential Equations, 23:3 (1987), 300-305.

9. А. С. Юдина, "О методе регуляризации для уравнений с регулярной особой точкой”, Тр. МЭИ, 357 (1978), 119-121.

10. А. С. Юдина, "Асимптотика решения сингулярного уравнения с регулярной особой точкой”, Тр. МЭИ, 566 (1982), 82-86.

11. А. С. Юдина, "Регуляризованные асимптотические разложения функций Бесселя", Тр. МЭИ, 573 (1982), 106-111.

12. С. А. Ломов, А. С. Юдина, "Структура фундаментальной системы решений сингулярно возмущенного уравнения с регулярной особой точкой”, Изв. АН $C C C P$. Сер. матем., 46:5 (1982), 1124-1133; англ. пер.: S. A. Lomov, A. S. Yudina, "The structure of a fundamental system of solutions of a singularly perturbed equation with a regular singular point", Math. USSR-Izv., 21:2 (1983), 415-424.

13. А. С. Юдина, "Асимптотическое решение задачи Коши для неоднородного уравнения Бесселя с мнимым параметром”, Сб. науч. трудов МЭИ, 141 (1987), 105-109.

14. Н. Г. де Брейн, Асимптотические методы в анализе, ИЛ, М., 1961; пер. с англ.: N. G. de Bruijn, Asymptotic methods in analysis, North-Holland, Amsterdam, 1958.

15. А. Эрдейи, Асимптотические разложения, Физматгиз, М., 1962; пер. с англ.: A. Erdelyi, Asymptotic expansions, Dover, New York, 1956.

A. С. Юдина (А. S. Yudina)

Поступило в редакцию

E-mail: a.s.yudina@mail.ru 